Pacific

Journal of

Mathematics

DEGENERATE FLAG VARIETIES AND SCHUBERT VARIETIES:

A CHARACTERISTIC FREE APPROACH

Giovanni Cerulli Irelli, Martina Lanini And Peter LitTelmann 


\title{
DEGENERATE FLAG VARIETIES AND SCHUBERT VARIETIES: A CHARACTERISTIC FREE APPROACH
}

\author{
Giovanni Cerulli Irelli, Martina LAnini And Peter Littelmann
}

\begin{abstract}
We consider the PBW filtrations over $\mathbb{Z}$ of the irreducible highest weight modules in type $A_{n}$ and $C_{n}$. We show that the associated graded modules can be realized as Demazure modules for group schemes of the same type and doubled rank. We deduce that the corresponding degenerate flag varieties are isomorphic to Schubert varieties in any characteristic.
\end{abstract}

\section{Introduction}

Introduced by Evgeny Feigin in 2010, degenerate flag varieties naturally arise from a representation theoretic context. In fact, given a finite dimensional, highest weight irreducible module $V(\lambda)$ for a simple finite dimensional, complex Lie algebra, the corresponding degenerate flag variety $\mathcal{F} \ell(\lambda)^{a}$ is the closure of a certain highest weight orbit in the projectivization of $V(\lambda)^{a}$, a degenerate version of $V(\lambda)$.

If the algebra one starts with is of type $\mathrm{A}_{n}$ or $\mathrm{C}_{n}$, it was shown in [Cerulli Irelli and Lanini 2015] that, surprisingly, degenerate flag varieties can be realized as Schubert varieties in a partial flag variety of the same type and bigger rank. It is hence natural to ask whether also the modules $V(\lambda)^{a}$ are isomorphic to some already investigated objects. The aim of this paper is to address such a question and provide a positive answer to it. Feigin's degeneration procedure can be carried out over $\mathbb{Z}-$ see [Feigin et al. 2013] — and it is in this generality that we decided to approach the problem.

Our main theorem is the realization of $V(\lambda)^{a}$ as a Demazure module for a group scheme of the same type and doubled rank. This fact allows us to recover, as a corollary, the above-mentioned realization of $\mathcal{F} \ell(\lambda)^{a}$ as a Schubert variety. While the arguments in [Cerulli Irelli and Lanini 2015] relied on a linear algebraic description of the degenerate flag variety due to Feigin [2012], the proof we obtain here only uses the definition of $\mathcal{F} \ell(\lambda)^{a}$ as a closure of a highest weight orbit; hence it is more conceptual.

In what follows, we describe in more detail the main results of this article.

For simplicity let us start with the complex algebraic group $\mathrm{SL}_{n}(\mathbb{C})$ and its Lie algebra $\mathfrak{g}=\mathfrak{s l}_{n}$. We fix a Cartan decomposition $\mathfrak{g}=\mathfrak{n}^{-} \oplus \mathfrak{h} \oplus \mathfrak{n}^{+}$, where $\mathfrak{n}$ is

MSC2010: primary 17B10; secondary 17B35, 16S30, 20G40, 14N15.

Keywords: Schubert varieties, degenerate flag varieties, Demazure modules. 
the subalgebra of strictly upper triangular matrices, $\mathfrak{h}$ is the Cartan subalgebra consisting of diagonal matrices and $\mathfrak{n}^{-}$is the subalgebra of strictly lower triangular matrices. Let $\mathfrak{b}=\mathfrak{h} \oplus \mathfrak{n}^{+}$be the corresponding Borel subalgebra of $\mathfrak{g}$ and let $B \subset G$ be the Borel subgroup with Lie algebra $\mathfrak{b}$.

We use the notation $\tilde{B}, \tilde{\mathfrak{b}}, \tilde{\mathfrak{n}}^{+}, \tilde{\mathfrak{h}}$, and $\tilde{\mathfrak{n}}^{-}$for the corresponding subgroup of $\tilde{G}=\mathrm{SL}_{2 n}(\mathbb{C})$ and subalgebras of $\tilde{\mathfrak{g}}=\mathfrak{s l}_{2 n}$. Let $\mathfrak{n}^{-, a} \subset \mathfrak{s l}_{2 n}$ and $N^{-, a} \subseteq \mathrm{SL}_{2 n}(\mathbb{C})$ be the following commutative Lie subalgebra and commutative unipotent subgroup, respectively:

$$
\mathfrak{n}^{-, a}:=\left\{\left(\begin{array}{cc}
0 & N \\
0 & 0
\end{array}\right) \in \mathfrak{s l}_{2 n} \mid N \in \mathfrak{n}^{-}\right\}, \quad N^{-, a}:=\left\{\left(\begin{array}{cc}
\text { II } & N \\
0 & \text { II }
\end{array}\right) \in \mathrm{SL}_{2 n} \mid N \in \mathfrak{n}^{-}\right\} .
$$

We view $\mathfrak{n}^{-, a}$ as the abelianization of $\mathfrak{n}^{-}$, i.e., we have the canonical vector space isomorphism between the two vector spaces, but $\mathfrak{n}^{-, a}$ is endowed with the trivial Lie bracket. The enveloping algebra of $\mathfrak{n}^{-, a}$ is $S^{\bullet}\left(\mathfrak{n}^{-, a}\right)$. The embedding $\mathfrak{n}^{-, a} \hookrightarrow \tilde{\mathfrak{b}}$ induces an embedding $S^{\bullet}\left(\mathfrak{n}^{-, a}\right) \hookrightarrow U(\tilde{\mathfrak{b}})$, so any $U(\tilde{\mathfrak{b}})$-module inherits in a natural way the structure of a $S^{\bullet}\left(\mathfrak{n}^{-, a}\right)$-module.

A well investigated class of $U(\tilde{\mathfrak{b}})$-modules are the Demazure modules: let $\mu$ be a dominant integral weight for $\tilde{\mathfrak{g}}$ and let $\tilde{V}(\mu)$ be the corresponding irreducible representation. For an element $w$ of the Weyl group $\tilde{W}$ of $\tilde{\mathfrak{g}}$, the weight space $\tilde{V}(\mu)_{w \mu}$ of weight $w \mu$ is one-dimensional; fix a generator $v_{w \mu}$. Recall that the Demazure submodule $\tilde{V}(\mu)_{w}$ is by definition the cyclic $U(\tilde{\mathfrak{b}})$-module generated by $v_{w \mu}$, i.e., $\tilde{V}(\mu)_{w}=U(\tilde{\mathfrak{b}}) . v_{w \mu}$, and the Schubert variety $X(w)$ is the closure of the orbit $\tilde{B} .\left[v_{w \mu}\right] \subseteq \mathbb{P}(\tilde{V}(\mu))$.

A special class of $S^{\bullet}\left(\mathfrak{n}^{-, a}\right)$-modules has been investigated in [Feigin et al. 2011a; $2011 b]$. Let $\lambda$ be a dominant integral weight for $\mathfrak{g}$, let $V(\lambda)$ be the corresponding irreducible representation and fix a highest weight vector $v_{\lambda}$. The PBW filtration on $U\left(\mathfrak{n}^{-}\right)$induces a filtration on the cyclic $U\left(\mathfrak{n}^{-}\right)$-module $V(\lambda)=U\left(\mathfrak{n}^{-}\right) \cdot v_{\lambda}$, and the associated graded space $V^{a}(\lambda):=\operatorname{gr} V(\lambda)$ becomes a module for the associated graded algebra $S^{\bullet}\left(\mathfrak{n}^{-}\right):=\operatorname{gr} U\left(\mathfrak{n}^{-}\right) \simeq S^{\bullet}\left(\mathfrak{n}^{-, a}\right)$.

The action of $\mathfrak{n}^{-, a}$ on $V^{a}(\lambda)$ can be integrated to an action of $N^{-, a}$. In analogy with the classical case we call the closure of the orbit $\mathcal{F}_{\lambda}^{a}:=\overline{N^{-, a} \cdot\left[v_{\lambda}\right]} \subseteq \mathbb{P}\left(V^{a}(\lambda)\right)$ the degenerate flag variety.

The aim of this article is to connect these two constructions and extend the results in [Cerulli Irelli and Lanini 2015] to an algebraically closed field $k$ of arbitrary characteristic. In fact, the results hold even over $\mathbb{Z}$. For simplicity, we formulate them in the introduction for an algebraically closed field $k$. In the following, we consider the case $G=\mathrm{SL}_{n}(k)$ and $\tilde{G}=\mathrm{SL}_{2 n}(k)$, respectively $G=\mathrm{Sp}_{2 m}(k)$, and $\tilde{G}=\mathrm{Sp}_{4 m}(k)$, and we replace the irreducible module of highest weight $\lambda$ by the Weyl module of highest weight $\lambda$, using the same notation $V(\lambda)$. For the precise description of the highest weight $\Psi(\lambda)$, see Definitions 2.1 and 5.3; for a 
description of the Weyl group element $\tau \in \widetilde{W}$, see Definitions 2.2 and 5.1; and for the construction of the Lie algebra $\mathfrak{n}^{-, a}$ in the symplectic case, see Section 5. For a dominant $G$-weight $\lambda$ let $\lambda^{*}$ be the dual dominant weight, so for the symplectic case we have $\lambda=\lambda^{*}$, and in the $\mathrm{SL}_{n}$ case we have $\lambda^{*}=\sum_{i=1}^{n-1} m_{i} \omega_{n-i}$ for $\lambda=\sum_{i=1}^{n-1} m_{i} \omega_{i}$ in the notation as in [Bourbaki 1968].

Theorem. Let $\lambda$ be a dominant $G$-weight.

(i) The Demazure submodule $\tilde{V}_{k}\left(\Psi\left(\lambda^{*}\right)\right)_{\tau}$ of the $\tilde{G}$-module $\tilde{V}_{k}\left(\Psi\left(\lambda^{*}\right)\right)$ is isomorphic, as an $\mathfrak{n}^{-, a}$-module, to the abelianized module $V^{a}(\lambda)$.

(ii) The Schubert variety $X(\tau) \subset \mathbb{P}\left(\tilde{V}\left(\Psi\left(\lambda^{*}\right)\right)_{\tau}\right)$ is isomorphic to the degenerate flag variety $\mathcal{F}^{a}(\lambda)$, and this isomorphism induces an $S^{\bullet}\left(\mathfrak{n}^{-, a}\right)$-module isomorphism

$$
H^{0}\left(X(\tau), \mathcal{L}_{\Psi\left(\lambda^{*}\right)}\right) \simeq\left(V^{a}(\lambda)\right)^{*} .
$$

Using the isomorphism above, we deduce the defining relations for $V^{a}(\lambda)$ from the defining relations of the Demazure module. Translated back into the language of the abelianized algebras we get the following: in the $\mathrm{SL}_{n}$ case, let $R^{++}=R^{+}$ be the set of positive roots, and in the symplectic case, set

$$
R^{++}=\left\{\epsilon_{i}-\epsilon_{j} \mid 1 \leq i<j \leq m\right\} \cup\left\{2 \epsilon_{i} \mid 1 \leq i \leq m\right\} .
$$

Corollary. The abelianized module $V^{a}(\lambda)$ is isomorphic as a cyclic $S^{\bullet}\left(\mathfrak{n}^{-, a}\right)$ module to $S^{\bullet}\left(\mathfrak{n}^{-, a}\right) / I(\lambda)$, where $I(\lambda)$ is the ideal:

$$
I(\lambda)=S^{\bullet}\left(\mathfrak{n}^{-, a}\right)\left(U\left(\mathfrak{n}^{+}\right) \circ \operatorname{span}\left\{f_{\alpha}^{\left(\left\langle\lambda, \alpha^{\vee}\right\rangle+1\right)} \mid \alpha \in R^{++}\right\}\right) \subseteq S^{\bullet}\left(\mathfrak{n}^{-, a}\right) .
$$

The identification of the degenerate flag variety as a Schubert variety implies the following corollary immediately; see also [Feigin and Finkelberg 2013; Feigin et al. 2014].

Corollary. The degenerate flag variety $\mathcal{F}^{a}(\lambda)$ is projectively normal, and it has rational singularities.

\section{Some special commutative unipotent subgroups}

Let $k$ be a field. Given a subspace $\mathfrak{N} \subseteq M_{n}(k)$ and a vector space automorphism $\eta: \mathfrak{N} \rightarrow \mathfrak{N}$, denote by $\mathfrak{N}_{\eta}^{a} \subseteq M_{2 n}(k)$ respectively $N_{\eta}^{a} \subseteq \mathrm{GL}_{2 n}(k)$ the following commutative nilpotent Lie subalgebra of $M_{2 n}(k)$, respectively commutative unipotent subgroup of $\mathrm{GL}_{2 n}(k)$ :

$$
\mathfrak{N}_{\eta}^{a}:=\left\{\left(\begin{array}{cc}
0 & \eta(A) \\
0 & 0
\end{array}\right) \mid A \in \mathfrak{N}\right\}, \quad N_{\eta}^{a}:=\left\{\left(\begin{array}{cc}
\mathbb{1} & \eta(A) \\
0 & \mathbb{1}
\end{array}\right) \mid A \in \mathfrak{N}\right\} .
$$

If $\mathfrak{N} \subseteq M_{n}(k)$ is a Lie subalgebra, then we think of $\mathfrak{N}_{\eta}^{a}$ as an abelianized version of $\mathfrak{N}$. Similarly one may think of $N_{\eta}^{a}$ as an abelianized version of a subgroup $N \subseteq \mathrm{GL}_{2 n}(k)$. We will be more precise in the following examples. 
Example 1.1. Let $k$ be an algebraically closed field of characteristic zero. We fix as a maximal torus $T \subset \mathrm{SL}_{n}$ the subgroup of diagonal matrices, and let $B$ be the Borel subgroup of upper triangular matrices. Let us denote by $\mathfrak{s l}_{n}, \mathfrak{b}$, and $\mathfrak{h}$ the corresponding Lie algebras and let $\mathfrak{g}=\mathfrak{n}^{-} \oplus \mathfrak{h} \oplus \mathfrak{n}^{+}$be the Cartan decomposition. The choice of a maximal torus and a Borel subgroup as above determines the set of positive roots $\Phi^{+}$and hence, according to the adjoint action of $\mathfrak{h}$, the root space decomposition $\mathfrak{n}^{-}=\bigoplus_{\alpha \in \Phi^{+}} \mathfrak{n}_{-\alpha}^{-}$. In this example we set $\mathfrak{N}=\mathfrak{n}^{-}$, and $N=U^{-}$is the unipotent radical of the opposite Borel subgroup $B^{-}$. The map $\eta$ is the identity map, so we just omit it. Henceforth, we write $\mathfrak{n}^{-, a}$ for $\mathfrak{N}^{a} \subset \mathfrak{s l}_{2 n}(k)$ and $N^{-, a}$ for $N^{a} \subset \mathrm{SL}_{2 n}$.

Note that $\mathfrak{n}^{-, a} \subset \mathfrak{s l}_{2 n}$ is a Lie subalgebra of the Borel subalgebra $\tilde{\mathfrak{b}} \subset \mathfrak{s l}_{2 n}$ and $N^{-, a}$ is an abelian subgroup of the Borel subgroup $\tilde{B} \subset \mathrm{SL}_{2 n}$ (of upper triangular matrices). We can think of $N^{-, a}$ as an abelianized version of $U^{-}$.

The subgroup $N^{-, a}$, as well as the Lie algebra $\mathfrak{n}^{-, a}$, is stable under conjugation with respect to the maximal torus $\tilde{T} \subset \mathrm{SL}_{2 n}$, where $\tilde{T} \subset \mathrm{SL}_{2 n}$ consists of the diagonal matrices. The group $N^{-, a}$ hence decomposes as a product of root subgroups of the group $\mathrm{SL}_{2 n}$, and $\mathfrak{n}^{-, a}$ decomposes into the direct sum of root subspaces for the Lie algebra $\mathfrak{s l}_{2 n}$. We get an induced map $\phi: \Phi^{+} \rightarrow \tilde{\Phi}^{+}$between the set of positive roots of $\mathfrak{s l}_{n}$ and the positive roots of $\mathfrak{s l}_{2 n}$, such that $\mathfrak{n}^{-, a}=\bigoplus_{\alpha \in \Phi^{+}} \mathfrak{n}_{\phi(\alpha)}^{-, a}$.

Example 1.2. Let $k$ be an algebraically closed field of characteristic 0. Let $\left\{e_{1}, \ldots, e_{2 n}\right\}$ be the canonical basis of $k^{2 n}$, and fix a nondegenerate skew symmetric form by the conditions $\left\langle e_{i}, e_{j}\right\rangle=\delta_{j, 2 n-i+1}=-\left\langle e_{j}, e_{i}\right\rangle$ for $1 \leq i \leq n, 1 \leq j \leq 2 n$. Let $\mathrm{Sp}_{2 m}$ be the associated symplectic group. By the choice of the form we can fix as a Borel subgroup $B$ the subgroup of upper triangular matrices in $\mathrm{Sp}_{2 m}$ and let $T$ be its maximal torus consisting of diagonal matrices. Let us denote by $\mathfrak{s p}_{2 m}, \mathfrak{b}$, and $\mathfrak{h}$ the corresponding Lie algebras and let $\mathfrak{g}=\mathfrak{n}^{-} \oplus \mathfrak{h} \oplus \mathfrak{n}^{+}$be the Cartan decomposition.

The choice of the torus and the Borel subgroup as above determines a set of positive roots $\Phi^{+}$and hence, according to the adjoint action of $\mathfrak{h}$, the root space decomposition $\mathfrak{n}^{-}=\bigoplus_{\alpha \in \Phi^{+}} \mathfrak{n}_{-\alpha}^{-}$. In this example we set $\mathfrak{N}=\mathfrak{n}^{-}$, and $N=U^{-}$ is the unipotent radical of the opposite Borel subgroup $B^{-}$. Let $\eta: \mathfrak{n}^{-} \rightarrow \mathfrak{n}^{-}$be the linear map sending a matrix $\left(m_{i, j}\right)_{1 \leq i, j \leq 2 n}$ to the matrix $\left(m_{i, j}^{\prime}\right)_{1 \leq i, j \leq 2 n}$, where $m_{i, j}^{\prime}=m_{i, j}$ if $i \leq n$ or $j \leq n$ and $m_{i, j}^{\prime}=-m_{i, j}$ if both indices are strictly larger than $n$. We write henceforth $\mathfrak{n}_{\eta}^{-, a}$ for $\mathfrak{N}_{\eta}^{a} \subset \mathfrak{s p}_{4 n}(k)$ and $N_{\eta}^{-, a}$ for $N_{\eta}^{a} \subset \mathrm{Sp}_{4 n}$.

Note that $\mathfrak{n}_{\eta}^{-, a} \subset \mathfrak{s p}_{4 n}$ is a Lie subalgebra of the Borel subalgebra (of upper triangular matrices) $\tilde{\mathfrak{b}} \subset \mathfrak{s p}_{4 n}$ and $N_{\eta}^{-, a}$ is an abelian subgroup of the Borel subgroup $\tilde{B} \subset \mathrm{Sp}_{4 n}$ (of upper triangular matrices). We can think of $N_{\eta}^{-, a}$ as an abelianized version of $U^{-}$.

The subgroup $N_{\eta}^{-, a}$ is stable under conjugation with respect to the maximal torus $\tilde{T} \subset \mathrm{Sp}_{4 n}$, where $\tilde{T} \subset \mathrm{Sp}_{4 n}$ consists of the diagonal matrices. The group $N_{\eta}^{-, a}$ hence decomposes as a product of root subgroups of the group $\mathrm{Sp}_{4 n}$ and $\mathfrak{n}^{-, a}$ decomposes into the direct sum of root subspaces for the Lie algebra $\mathfrak{s p}_{4 n}$. We get an induced 
map $\phi: \Phi^{+} \rightarrow \tilde{\Phi}^{+}$between the set of positive roots of $\mathfrak{s p}_{2 n}$ and the positive roots of $\mathfrak{s p}_{4 n}$, such that $\mathfrak{n}_{\eta}^{-, a}=\bigoplus_{\alpha \in \Phi^{+}} \mathfrak{n}_{\phi(\alpha), \eta}^{-, a}$.

Example 1.3. To get a characteristic free approach for $G$ as above, let $G_{\mathbb{Z}}$ be a split and connected simple algebraic $\mathbb{Z}$-group of type $\mathrm{A}_{n}$ or $\mathrm{C}_{\mathrm{n}}$. For any commutative ring $A$ set $G_{A}=\left(G_{\mathbb{Z}}\right)_{A}$, and for a field set $G=G_{k}$, for this and the following; see also [Jantzen 1987]. Then $G_{k}$ is for any algebraically closed field a reduced $k$-group, and it is connected and reductive. Its $\operatorname{Lie}$ algebra $\operatorname{Lie}\left(G_{\mathbb{Z}}\right)$ is a free $\mathrm{Lie}$ algebra of finite rank and Lie $G_{k}=\operatorname{Lie}\left(G_{\mathbb{Z}}\right) \otimes_{\mathbb{Z}} k$. Let $T_{\mathbb{Z}} \subset G_{\mathbb{Z}}$ be a split maximal torus and set $T_{A}=\left(T_{\mathbb{Z}}\right)_{A}$ for any ring $A$ and $T=T_{k}$. We have a root space decomposition Lie $G=\operatorname{Lie} T \oplus \bigoplus_{\alpha \in \Phi}(\text { Lie } G)_{\alpha}$ where $(\text { Lie } G)_{\alpha}=\left(\text { Lie } G_{\mathbb{Z}}\right)_{\alpha} \otimes_{\mathbb{Z}} k$, and corresponding root subgroups (defined over $\mathbb{Z}) x_{\alpha}: \mathbb{G}_{a} \rightarrow G$ such that the tangent map $\mathrm{d} x_{\alpha}$ induces an isomorphism between the Lie algebra of the additive group $\mathbb{G}_{a}$ and (Lie $\left.G\right)_{\alpha}$. The functor which associates to any commutative $\operatorname{ring} A$ the group $x_{\alpha}\left(\mathbb{G}_{a}(A)\right)=x_{\alpha}(A)$ is a closed subgroup of $G$ denoted by $U_{\alpha}$, and we have $\operatorname{Lie}\left(U_{\alpha}\right)=(\operatorname{Lie} G)_{\alpha}$. Over $\mathbb{Z}$ we denote the corresponding subgroup by $U_{\alpha, \mathbb{Z}}$, and over a field $k$ we have $U_{\alpha}=\left(U_{\alpha, \mathbb{Z}}\right)_{k}$.

The construction described in Examples 1.1 and 1.2 makes (in this language) sense over $\mathbb{Z}$ or over any field. As before, let $\tilde{G}$ be the group of the same type but twice the rank, we denote the corresponding Borel subgroup, maximal torus, etc. by $\tilde{B}, \tilde{T}$, etc. The construction in the examples above associates to every root $\alpha \in \Phi$ a root $\phi(\alpha)$ in the root system of $\tilde{G}$. For the $\mathbb{Z}$-group $G_{\mathbb{Z}}$ we have the subgroup $U_{\mathbb{Z}}^{-}$and the Lie algebra $\mathfrak{n}_{\mathbb{Z}}^{-}=\bigoplus_{\alpha \in \Phi^{+}} \mathfrak{n}_{\mathbb{Z},-\alpha}^{-}$, and we associate to this pair a new pair given by a commutative subgroup $N_{\eta}^{-, a}$ of the $\mathbb{Z}$-group $\tilde{G}_{\mathbb{Z}}$ and an abelian Lie algebra $\mathfrak{n}_{\mathbb{Z}, \eta}^{-, a}$. The first is the subgroup of the Borel subgroup $\tilde{B}_{\mathbb{Z}} \subset \tilde{G}_{\mathbb{Z}}$ generated by the commuting root subgroups $U_{\phi(\alpha), \mathbb{Z}}, \alpha \in \Phi^{+}$, and the second is the abelian Lie algebra $\mathfrak{n}_{\mathbb{Z}, \eta}^{-, a}=\bigoplus_{\alpha \in \Phi^{+}}(\text {Lie } G)_{\mathbb{Z}, \phi(\alpha)}^{\sim}$ given as the sum of root subalgebras.

\section{A special Schubert variety: the $\mathrm{SL}_{n}$ case}

We want to realize in the situation of Example 1.1 the abelianized representation $V(\lambda)^{a}$ for $N_{\eta}^{-, a}$ as a Demazure submodule of an irreducible representation for the group $\mathrm{SL}_{2 n}$.

2A. A special Weyl group element. Let $\widetilde{W}$ be the Weyl group of $\mathrm{SL}_{2 n}(\mathbb{C})$; it is the symmetric group $\mathcal{S}_{2 n}$ generated by the transpositions $s_{i}, i=1, \ldots, 2 n-1$. Let $\mathfrak{h} \subset \mathfrak{g}=\mathfrak{s l}_{n}$ (respectively, $\tilde{\mathfrak{h}} \subset \tilde{\mathfrak{g}}=\mathfrak{s l}_{2 n}$ ) be the Cartan subalgebra of traceless complex diagonal matrices. For an element $\alpha \in \mathfrak{h}^{*}$ and an element $h \in \mathfrak{h}$ we denote by $\langle h, \alpha\rangle$ the evaluation of $\alpha$ in $h$. Let $\left\{\epsilon_{1}, \ldots, \epsilon_{n}\right\}$ be the elements of the dual vector space $\mathfrak{h}^{*}$ such that $\left\langle h, \epsilon_{i}\right\rangle$ is the $i$-th entry in the diagonal matrix $h \in \mathfrak{h}$. We use the same notation $\langle\tilde{h}, \tilde{\alpha}\rangle$ for elements $\tilde{h} \in \tilde{\mathfrak{h}}$ and $\tilde{\alpha} \in \tilde{\mathfrak{h}}^{*}$, and the linear forms $\left\{\tilde{\epsilon}_{1}, \ldots, \tilde{\epsilon}_{2 n}\right\}$ in $\tilde{\mathfrak{h}}^{*}$ are defined as above. 
The roots of $\mathfrak{g}$ (resp., $\tilde{\mathfrak{g}}$ ) are the elements $\alpha_{i, j}:=\epsilon_{i}-\epsilon_{j}\left(\right.$ resp., $\left.\tilde{\alpha}_{i, j}=\tilde{\epsilon}_{i}-\tilde{\epsilon}_{j}\right)$ for $i \neq j$. We choose as a Borel subalgebra of $\mathfrak{g}$ the subalgebra $\mathfrak{b}$ of upper triangular matrices. The corresponding simple roots are $\alpha_{1}, \cdots, \alpha_{n-1}$ given by $\alpha_{i}:=\alpha_{i, i+1}$. For every root $\alpha$, we denote by $\alpha^{\vee}$ its coroot: this is the unique element of $\mathfrak{h}$ such that the reflection $s_{\alpha} \in \mathfrak{h}^{*}$ along $\alpha$ acts as $s_{\alpha}(\lambda)=\lambda-\left\langle\alpha^{\vee}, \lambda\right\rangle \alpha$. Moreover we denote by $E_{\alpha}$ the corresponding root vector. We denote by $\omega_{i}=\epsilon_{1}+\cdots+\epsilon_{i}$ (resp., $\tilde{\omega}_{i}=$ $\left.\tilde{\epsilon}_{1}+\cdots+\tilde{\epsilon}_{i}\right)$ the $i$-th fundamental weight of $\mathfrak{g}($ resp., $\tilde{\mathfrak{g}})$, where $i=1,2, \ldots, n-1$ (resp., $i=1, \ldots, 2 n-1)$. They are characterized by the property $\left\langle\alpha_{i}^{\vee}, \omega_{j}\right\rangle=\delta_{i, j}$.

Definition 2.1. Let $\Psi: \mathfrak{h}^{*} \rightarrow \tilde{\mathfrak{h}}^{*}$ be the linear map defined on the weight lattice by

$$
\Psi\left(\sum_{i=1}^{n-1} a_{i} \omega_{i}\right):=\sum_{i=1}^{n-1} a_{i} \tilde{\omega}_{2 i} .
$$

Note that $\Psi$ sends dominant weights to dominant weights. For every fundamental weight $\tilde{\omega}_{k}$, we denote the corresponding parabolic subgroup by $P_{\tilde{\omega}_{k}}$ and by $\tilde{W}_{\tilde{\omega}_{k}}$ the corresponding subgroup of $\tilde{W}$ which is the Weyl group of the semisimple part of $P_{\tilde{\omega}_{k}}$. Note that $\tilde{W}_{\tilde{\omega}_{k}}$ is generated by all the simple transpositions $s_{i}$ but $s_{k}$. Let $\rho=\omega_{1}+\cdots+\omega_{n-1}$. Then $\Psi(\rho)=\tilde{\omega}_{2}+\tilde{\omega}_{4}+\cdots+\tilde{\omega}_{2 n-2}$. The parabolic subgroup $Q=P_{\tilde{\omega}_{2}+\cdots+\tilde{\omega}_{2 n-2}}$ which is the stabilizer of $\Psi(\rho)$ will play an important role. The Weyl group of the semisimple part of $Q$ is denoted by $\tilde{W}^{J}$.

Definition 2.2. We define in the Weyl group $\widetilde{W}$ the element $\tau$ by

$$
\tau=\left(s_{n} s_{n+1} \cdots s_{2 n-3} s_{2 n-2}\right)\left(s_{n-1} s_{n} \cdots s_{2 n-4}\right) \cdots\left(s_{4} s_{5} s_{6}\right)\left(s_{3} s_{4}\right) s_{2} .
$$

It is easy to see that the decomposition is reduced and $\tau$ is a minimal length representative in its class in $\widetilde{W} / \tilde{W}^{J}$. Another description of $\tau$ can be given by viewing $\tau$ as a permutation of the set $\{1, \ldots, 2 n\}$ :

$$
\tau(t)=\left\{\begin{array}{cl}
n+k & \text { if } t=2 k \\
k & \text { if } t=2 k-1,
\end{array}\right.
$$

for $k=1,2, \ldots, n$. It follows now immediately from (3):

Lemma 2.3. In the irreducible $\mathrm{SL}_{2 n}(\mathbb{C})$-representation $\tilde{V}\left(\tilde{\omega}_{2 i}\right)=\wedge^{2 i} \mathbb{C}^{2 n}$ let $v_{0}$ be the highest weight vector $v_{0}=e_{1} \wedge e_{2} \wedge \cdots \wedge e_{2 i}$. Then (up to a sign),

$$
\tau\left(v_{0}\right)=v_{\tau}=e_{1} \wedge e_{2} \wedge \cdots \wedge e_{i} \wedge e_{n+1} \wedge e_{n+2} \wedge \cdots \wedge e_{n+i} .
$$

Let $\lambda=b_{1} \epsilon_{1}+\cdots+b_{n-1} \epsilon_{n-1}$, with $b_{1} \geq \cdots \geq b_{n-1} \geq 0$, be a dominant weight for $\mathrm{SL}_{n}(\mathbb{C})$. The next result follows directly from Lemma 2.3.

Lemma 2.4. $\tau(\Psi(\lambda))=b_{1} \tilde{\epsilon}_{1}+\cdots+b_{n-1} \tilde{\epsilon}_{n-1}+b_{1} \tilde{\epsilon}_{n+1}+\cdots+b_{n-1} \tilde{\epsilon}_{2 n-1}$. 
In Example 1.1 we have introduced a map $\phi: \Phi^{+} \rightarrow \tilde{\Phi}^{+}$between the positive roots of $\mathfrak{s l}_{n}$ and the positive roots of $\mathfrak{s l}_{2 n}$. Note that the image of $\alpha=\epsilon_{i}-\epsilon_{j}$, $1 \leq i<j \leq n$ is the $\operatorname{root} \phi(\alpha)=\tilde{\epsilon}_{j}-\tilde{\epsilon}_{n+i}$.

Lemma 2.5. (i) Let $\lambda$ be a dominant weight for $\mathrm{SL}_{n}(\mathbb{C})$, and let $\tilde{\alpha}$ be a positive $\mathrm{SL}_{2 n}$-root. Then $\left\langle\tilde{\alpha}^{\vee}, \tau(\Psi(\lambda))\right\rangle<0$ only if the root space of $\tilde{\alpha}$ lies in $\mathfrak{n}^{-, a}$.

(ii) Let $\lambda$ be a dominant $\mathrm{SL}_{n}$-weight, and let $\alpha=\epsilon_{i}-\epsilon_{j}$ be a positive $\mathrm{SL}_{n}$-root. Then

$$
\left\langle\alpha^{\vee}, \lambda\right\rangle=-\left\langle\phi(\alpha)^{\vee}, \tau(\Psi(\lambda))\right\rangle .
$$

(iii) Let $\lambda$ be a dominant weight for $\mathrm{SL}_{n}(\mathbb{C})$, and let $\tilde{\alpha}=\tilde{\epsilon}_{p}-\tilde{\epsilon}_{q}$ be a positive $\mathrm{SL}_{2 n}$-root. Then $E_{\tilde{\alpha}} v_{\tau} \neq 0$ in $\tilde{V}(\Psi(\lambda))$ only if $\tilde{\alpha}$ is of the form $\tilde{\alpha}=\tilde{\epsilon}_{j}-\tilde{\epsilon}_{n+i}$, $1 \leq i<j \leq n$ and $\left\langle\left(\epsilon_{i}-\epsilon_{j}\right)^{\vee}, \lambda\right\rangle>0$.

Proof. Let $\tilde{\alpha}=\tilde{\epsilon}_{i}-\tilde{\epsilon}_{j}$ be a positive root. By Lemma 2.4, for $\lambda=b_{1} \epsilon_{1}+\cdots+b_{n-1} \epsilon_{n-1}$, we get

$$
\left\langle\tilde{\alpha}^{\vee}, \tau(\Psi(\lambda))\right\rangle=\left\{\begin{aligned}
b_{i}-b_{j} \geq 0 & \text { if } 1 \leq i<j \leq n, \\
b_{i}-b_{j-n} \geq 0 & \text { if } 1 \leq i \leq n \text { and } n+i \leq j \leq 2 n, \\
b_{i}-b_{j-n} \leq 0 & \text { if } 1 \leq i \leq n \text { and } n+1 \leq j<n+i, \\
b_{i-n}-b_{j-n} \geq 0 & \text { if } n+1 \leq i<j \leq 2 n,
\end{aligned}\right.
$$

which proves the lemma.

The decomposition in (2) is reduced, but if we apply $\tau$ to a fundamental weight, then it is possible to omit some of the reflections. A simple calculation shows:

Lemma 2.6. Let $\tilde{\omega}_{2 i}$ be the $2 i$-th fundamental weight for $\mathrm{SL}_{2 n}(\mathbb{C})$. Then

$$
\tau\left(\tilde{\omega}_{2 i}\right)=\left(s_{n} s_{n+1} \cdots s_{n+i-1}\right) \cdots\left(s_{i+2} \cdots s_{2 i+1}\right)\left(s_{i+1} \cdots s_{2 i-1} s_{2 i}\right)\left(\tilde{\omega}_{2 i}\right) .
$$

Let $L(i)$ be the semisimple part of the Levi subgroup of $\mathrm{SL}_{2 n}(\mathbb{C})$ associated with the simple roots $\tilde{\alpha}_{i+1}, \tilde{\alpha}_{i+2}, \ldots, \tilde{\alpha}_{i+n-1}$, denote by $\mathfrak{l}(i)$ the Lie algebra of $L(i)$. Note that $L(i)$ is isomorphic to $\mathrm{SL}_{n}(\mathbb{C})$. Let $\varpi_{1}, \ldots, \varpi_{n-1}$ be the fundamental weights of $L(i)$, the enumeration is such that the simple root $\tilde{\alpha}_{i+j}$ of $L(i) \subseteq \mathrm{SL}_{2 n}(\mathbb{C})$ corresponds to $\varpi_{j}$.

The restriction of $\tilde{\omega}_{2 i}$ to $L(i)$ is $\varpi_{i}$. Let $W^{L(i)}$ be the Weyl group of $L(i)$, we can identify it with the subgroup of the Weyl group of $\mathrm{SL}_{2 n}$ generated by the reflections $s_{i+1}, s_{i+2}, \ldots, s_{i+n-1}$. Using Lemma 2.6, it is easy to see:

Lemma 2.7. A reduced decomposition of the longest word of $W^{L(i)}$ modulo the stabilizer $W_{\varpi_{i}}^{L(i)}$ of $\varpi_{i}$ in $W^{L(i)}$ is given by

$$
\left(s_{n} s_{n+1} \cdots s_{n+i-1}\right) \cdots\left(s_{i+2} \cdots s_{2 i+1}\right)\left(s_{i+1} \cdots s_{2 i-1} s_{2 i}\right) .
$$




\section{The fundamental representations: the $\mathfrak{s l}_{n}$ case}

We switch now to Lie algebras and hyperalgebras over $\mathbb{Z}$. Fix a Chevalley basis for the Lie algebra $\mathfrak{g}_{\mathbb{Z}}=\mathfrak{s l}_{n, \mathbb{Z}} \subset \mathfrak{s l}_{n, \mathbb{C}}$ :

$$
\left\{f_{\alpha}, e_{\alpha} \mid \alpha \in \Phi^{+}\right\} \cup\left\{h_{1}, \ldots, h_{n-1}\right\},
$$

where $f_{\alpha} \in \mathfrak{g}_{\mathbb{Z},-\alpha}, e_{\alpha} \in \mathfrak{g}_{\mathbb{Z}, \alpha}$, and $h_{i} \in \mathfrak{h}_{\mathbb{Z}}$. For any $m \in \mathbb{Z}_{\geq 1}$, we define the following elements in $U(\mathfrak{g})$ :

$$
e_{\alpha}^{(m)}=\frac{e_{\alpha}^{m}}{m !}, \quad f_{\alpha}^{(m)}=\frac{f_{\alpha}^{m}}{m !}, \quad\left(\begin{array}{c}
h_{i} \\
m
\end{array}\right)=\frac{h_{i}\left(h_{i}-1\right) \cdots\left(h_{i}-m+1\right)}{m},
$$

and for $m=0$ we set $e_{\alpha}^{(0)}=f_{\alpha}^{(0)}=\left(\begin{array}{c}h_{i} \\ 0\end{array}\right)=1$. Recall that the hyperalgebra $U_{\mathbb{Z}}\left(\mathfrak{s l}_{n}\right)$ of $\left(\mathrm{SL}_{n}\right)_{\mathbb{Z}}$ is the $\mathbb{Z}$-subalgebra of the complex enveloping algebra $U\left(\mathfrak{s l}_{n}\right)$ generated by the elements defined in (4). We will use capital letters to denote the Chevalley basis elements for $\mathfrak{s l}_{2 n, \mathbb{Z}}$ (e.g., $E_{\tilde{\alpha}}, F_{\tilde{\alpha}}, H_{i}$ ) and the generators of the hyperalgebra $U_{\mathbb{Z}}\left(\mathfrak{s l}_{2 n}\right)$ (e.g., $E_{\tilde{\alpha}}^{(m)}, F_{\tilde{\alpha}}^{(m)},\left(\begin{array}{c}H_{i} \\ m\end{array}\right)$ ). Similarly, let $U_{\mathbb{Z}}(\tilde{\mathfrak{b}})$ be the subalgebra generated by all $E_{\tilde{\alpha}}^{(m)}$ for $m \geq 0$ and $\tilde{\alpha}>0$, and all $\left(\begin{array}{c}H_{i} \\ m\end{array}\right)$ for $i=1, \ldots, 2 n-1$ and $m \geq 0$. Denote by $U_{\mathbb{Z}}(\mathfrak{l}(i))$ the hyperalgebra associated with $\mathfrak{l}(i)$, i.e., the subalgebra generated by all $F_{\tilde{\alpha}}^{(m)}, E_{\tilde{\alpha}}^{(m)}$ for $m \geq 0$ and $\tilde{\alpha}>0$, a root of the Levi subgroup $L(i)$, and by all $\left(\begin{array}{c}H_{j} \\ m\end{array}\right)$ for $j=i+1, \ldots, i+n-1$.

Let $\mu$ be a dominant integral weight for $\mathrm{SL}_{2 n}(\mathbb{C})$ and denote by $\tilde{V}(\mu)$ the irreducible $\mathrm{SL}_{2 n}(\mathbb{C})$-representation of highest weight $\mu$. Fix a highest weight vector $v_{\mu}$; the corresponding $\mathbb{Z}$-form is $\tilde{V}_{\mathbb{Z}}(\mu)=U_{\mathbb{Z}}\left(\mathfrak{s l}_{2 n}\right) v_{\mu}$. To define the Demazure module $\tilde{V}_{\mathbb{Z}}(\mu)_{w}$, fix a representative $\check{w}$ of $w$ in the simply connected Chevalley group associated with $\mathfrak{s l}_{2 n, \mathbb{Z}}$ and set $v_{w}:=\check{w}\left(v_{\mu}\right)$. The Demazure module $\tilde{V}_{\mathbb{Z}}(\lambda)_{w}$ is the cyclic $U_{\mathbb{Z}}(\tilde{\mathfrak{b}})$-subrepresentation $U_{\mathbb{Z}}(\tilde{\mathfrak{b}}) \cdot v_{w} \subseteq \tilde{V}_{\mathbb{Z}}(\mu)$.

Lemma 3.1. The Demazure module $\tilde{V}_{\mathbb{Z}}\left(\ell \tilde{\omega}_{2 i}\right)_{\tau}$ contained in $\tilde{V}_{\mathbb{Z}}\left(\ell \tilde{\omega}_{2 i}\right)$ is the Weyl module $V_{\mathbb{Z}}\left(\ell \varpi_{i}\right)$ of highest weight $\ell \varpi_{i}$ for $U_{\mathbb{Z}}(\mathfrak{l}(i))$.

Proof. Consider $\Psi\left(\ell \omega_{i}\right)=\ell \tilde{\omega}_{2 i}$ and recall that the restriction of $\tilde{\omega}_{2 i}$ to $l(i)$ is $\varpi_{i}$. So the $U_{\mathbb{Z}}(\mathfrak{l}(i))$-submodule $U_{\mathbb{Z}}(\mathfrak{l}(i)) v_{\ell \tilde{\omega}_{2 i}} \subseteq \tilde{V}_{\mathbb{Z}}\left(\ell \tilde{\omega}_{2 i}\right)$ is the Weyl module $V_{\mathbb{Z}}\left(\ell \varpi_{i}\right)$ of highest weight $\ell \varpi_{i}$ for $U_{\mathbb{Z}}(\mathfrak{l}(i))$. Let $U_{\mathbb{Z}}(\mathfrak{b}(i))$ be the subalgebra of $U_{\mathbb{Z}}(\mathfrak{l}(i))$ generated by the $E_{\tilde{\alpha}}^{(m)}$ for $m \geq 0$ and $\tilde{\alpha}>0$, a root of $\mathfrak{l}(i)$, and all $\left(\begin{array}{c}H_{j} \\ m\end{array}\right)$ for $j=$ $i+1, \ldots, i+n-1$.

The Weyl module $V_{\mathbb{Z}}\left(\ell \varpi_{i}\right)$ is a cyclic $U_{\mathbb{Z}}(\mathfrak{b}(i))$-module and is generated by a lowest weight vector of the form $\check{w}_{0, i}\left(v_{\ell \varpi_{i}}\right)$, where $\check{w}_{0, i}$ is an appropriate representative (in the Chevalley group associated with $\mathfrak{l}(i)$ ) of the longest element $w_{0, i}$ of the Weyl group $W^{L(i)}$ of $\mathfrak{l}(i)$. Recall that $W^{L(i)}$ can be identified with the subgroup of $\widetilde{W}$ generated by $s_{i+1}, \ldots, s_{n+i-1}$. Now

$$
\tilde{V}_{\mathbb{Z}}\left(\ell \tilde{\omega}_{2 i}\right)_{\tau}=U_{\mathbb{Z}}(\tilde{\mathfrak{b}}) v_{\tau}=U_{\mathbb{Z}}(\tilde{\mathfrak{b}}) v_{w_{0, i}}=U_{\mathbb{Z}}(\mathfrak{b}(i)) v_{w_{0, i}}=V_{\mathbb{Z}}\left(\ell \varpi_{i}\right) \subseteq \tilde{V}_{\mathbb{Z}}\left(\ell \tilde{\omega}_{2 i}\right)
$$


by Lemmas 2.5, 2.6, and 2.7.

The previous result implies in particular:

Corollary 3.2. $\operatorname{rank} \tilde{V}_{\mathbb{Z}}\left(\Psi\left(\ell \omega_{i}\right)\right)_{\tau}=\operatorname{rank} V_{\mathbb{Z}}\left(\ell \omega_{i}\right)$.

Let $\iota: \mathfrak{s l}_{n} \rightarrow \mathfrak{s l}_{n}$ be the Chevalley involution defined by $\left.\iota\right|_{\mathfrak{h}}=-1$ and that $\iota$ exchanges $e_{\alpha}$ and $-f_{\alpha}$. It follows that $\iota\left(\mathfrak{n}_{\mathbb{Z}}^{-}\right)=\mathfrak{n}_{\mathbb{Z}}^{+}$, and this map extends to an isomorphism of the corresponding hyperalgebras $\iota: U_{\mathbb{Z}}\left(\mathfrak{n}^{-}\right) \rightarrow U_{\mathbb{Z}}\left(\mathfrak{n}^{+}\right)$and the associated graded versions obtained via the PBW filtration: $\iota: S_{\mathbb{Z}}^{\bullet}\left(\mathfrak{n}^{-}\right) \rightarrow S_{\mathbb{Z}}^{\bullet}\left(\mathfrak{n}^{+}\right)$.

Let $\lambda=\sum a_{j} \omega_{j}$ be a dominant weight and set $\lambda^{*}:=\sum a_{j} \omega_{n-j}$. Fix a highest weight vector $v_{\lambda} \in V_{\mathbb{Z}}(\lambda)$ and a lowest weight vector $v_{w_{0}} \in V_{\mathbb{Z}}(\lambda)$, where $w_{0}$ is the longest word in the Weyl group of $\mathfrak{s l}_{n}$. We get two possible $S_{\mathbb{Z}}^{\bullet}\left(\mathfrak{n}^{-, a}\right)$-structures on $V_{\mathbb{Z}}(\lambda)$ : one uses the PBW filtration on $U_{\mathbb{Z}}\left(\mathfrak{n}^{-}\right)$to induce, via the highest weight vector, a PBW filtration on $V_{\mathbb{Z}}(\lambda)$ and passes to the associated graded module. One gets the module $V_{\mathbb{Z}}^{a}(\lambda)$ discussed before. Now one can do the same also for $U_{\mathbb{Z}}\left(\mathfrak{n}^{+}\right)$, once the highest weight vector is replaced by the lowest weight vector. We denote the cyclic $S_{\mathbb{Z}}^{\bullet}\left(\mathfrak{n}^{+}\right)$-module (generated by the lowest weight vector) by $V_{\mathbb{Z}}^{a,+}(\lambda)$. Now via $\iota$ this module also becomes naturally a $S_{\mathbb{Z}}^{\cdot}\left(\mathfrak{n}^{-}\right)$-module.

Lemma 3.3. As a $S_{\mathbb{Z}}^{\bullet}\left(\mathfrak{n}^{-}\right)$-module, $V_{\mathbb{Z}}^{a,+}(\lambda)$ is isomorphic to $V_{\mathbb{Z}}^{a}\left(\lambda^{*}\right)$.

Proof. Note that twisting the representation map with the Chevalley involution makes the lowest weight vector (the cyclic generator for the $U\left(\mathfrak{n}^{+}\right)$-action) into a cyclic generator for the $U\left(\mathfrak{n}^{-}\right)$-action. Recall that the Chevalley involution is equal to -1 on $\mathfrak{h}$, so after the twist this is now a highest weight vector of weight $\lambda^{*}=-w_{0}(\lambda)$, where $w_{0}$ is the longest word in $W$. Since the construction is compatible with the PBW filtrations with respect to the two algebras, the result for the associated graded modules follows immediately.

Proposition 3.4. As $S_{\mathbb{Z}}\left(\mathfrak{n}^{-, a}\right)$-modules, $\tilde{V}_{\mathbb{Z}}\left(\Psi\left(\ell \omega_{i}\right)\right)_{\tau} \simeq V_{\mathbb{Z}}^{a}\left(\ell \omega_{n-i}\right)$.

Proof. Let $\mathfrak{n}_{i}^{-, a} \subseteq \mathfrak{n}^{-, a}$ be the sum of all root subspaces of roots of the form $\tilde{\epsilon}_{k}-\tilde{\epsilon}_{n+\ell}$, where $1 \leq \ell \leq i \leq k \leq n$ and $\ell \neq k$. This is a commutative Lie subalgebra, which by Lemma 2.5 has the property

$$
V_{\mathbb{Z}}\left(\Psi\left(\ell \omega_{i}\right)\right)_{\tau}=U(\tilde{\mathfrak{b}}) \cdot v_{\tau}=U\left(\mathfrak{n}_{i}^{-, a}\right) \cdot v_{\tau}=S^{\bullet}\left(\mathfrak{n}_{i}^{-, a}\right) \cdot v_{\tau} .
$$

Since $\mathfrak{n}^{-, a}$ is commutative, all root vectors in $\mathfrak{n}^{-, a}$ which are not in $\mathfrak{n}_{i}^{-, a}$ act trivially on $V_{\mathbb{Z}}\left(\Psi\left(\ell \omega_{i}\right)\right)_{\tau}$.

Another way to describe $\mathfrak{n}_{i}^{-, a}$ is as the intersection $\mathfrak{n}^{-, a} \cap \mathfrak{l}(i)$. More precisely, this intersection is the nilpotent radical of the maximal parabolic subalgebra of $\mathfrak{l}(i)$ associated with the fundamental weight $\varpi_{n-i}$. By Lemma 3.1, we know that $V_{\mathbb{Z}}\left(\Psi\left(\ell \omega_{i}\right)\right)_{\tau}=U(\mathfrak{b}(i)) \cdot v_{\tau} \simeq V_{\mathbb{Z}}\left(\ell \varpi_{i}\right)$, and since $v_{\tau}$ is a lowest weight vector,

$$
V_{\mathbb{Z}}\left(\Psi\left(\ell \omega_{i}\right)\right)_{\tau}=U\left(\mathfrak{n}_{i}^{-, a}\right) \cdot v_{\tau} \simeq V_{\mathbb{Z}}\left(\ell \varpi_{i}\right) .
$$


Set $\mathfrak{n}^{+}(i)=\mathfrak{b}(i) \cap \tilde{\mathfrak{n}}^{+}$. By the isomorphism between $\mathfrak{l}(i)$ and $\mathfrak{s l}_{n}$ we can identify $\mathfrak{m}$ with $\mathfrak{n}^{+} \subset \mathfrak{s l}_{n}$. Consider the associated PBW filtration on $V_{\mathbb{Z}}\left(\ell \varpi_{i}\right)$ by applying the PBW filtration of $U\left(\mathfrak{n}^{+}(i)\right)$ to the lowest weight vector. Recall that after passing to the associated graded algebra $S^{\bullet}\left(\mathfrak{n}^{+}(i)\right)$, all root vectors not contained in $\mathfrak{n}_{i}^{-, a}$ act trivially on $V_{\mathbb{Z}}^{a,+}\left(\ell \varpi_{i}\right)$. Remember that we add a "+" to indicate that this is the associated graded space with respect to the filtration by the nilpotent radical of the fixed Borel subalgebra and not, as usual, of the opposite nilpotent algebra. Since $\varpi_{i}$ and $\varpi_{n-i}$ are cominuscule, $\mathfrak{n}_{i}^{-, a}$ is commutative and the PBW filtration on $V_{\mathbb{Z}}\left(\ell \varpi_{i}\right)$ is already a grading. It follows that the action of $\mathfrak{n}_{i}^{-, a}$ on $V_{\mathbb{Z}}\left(\ell \varpi_{i}\right)$ and $V_{\mathbb{Z}}^{a,+}\left(\ell \varpi_{i}\right)$ are the same, so the $\mathfrak{n}_{i}^{-, a}$ actions on $V_{\mathbb{Z}}^{a,+}\left(\ell \varpi_{i}\right)$ and $V_{\mathbb{Z}}\left(\Psi\left(\ell \omega_{i}\right)\right)_{\tau}$ are isomorphic; hence, so are the $\mathfrak{n}^{-, a}$ actions by trivial extension. The proposition follows now by Lemma 3.3.

\section{The general case for $\mathfrak{s l}_{n}$}

4A. We extend Proposition 3.4 to any dominant weight for $\mathfrak{s l}_{n}$. Recall that for a dominant weight $\lambda=a_{1} \omega_{1}+\cdots+a_{n-1} \omega_{n-1}$ we denote by $\lambda^{*}$ the dominant weight given by $\lambda^{*}=a_{n-1} \omega_{1}+\cdots+a_{1} \omega_{n-1}$.

Theorem 4.1. Let $\lambda$ be a dominant $\mathfrak{s l}_{n}$-weight. As an $\mathfrak{n}_{\mathbb{Z}}^{-, a}$-module, the Demazure

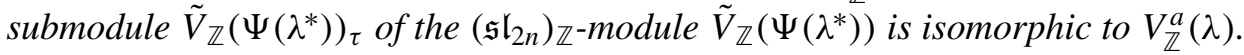

The proof of Theorem 4.1 will be given in Section 4G, and the strategy of proof is explained in Section 4C. We deduce a useful corollary.

Corollary 4.2. In particular, $V_{\mathbb{Z}}^{a}(\lambda)$ is free as a $\mathbb{Z}$-module.

Proof of the corollary. The Demazure module $\tilde{V}_{\mathbb{Z}}\left(\Psi\left(\lambda^{*}\right)\right)_{\tau}$ is a direct summand of the free $\mathbb{Z}$-module $\tilde{V}_{\mathbb{Z}}\left(\Psi\left(\lambda^{*}\right)\right)$ and hence free as $\mathbb{Z}$-module.

4B. The abelianized module $V_{\mathbb{Z}}^{a}(\lambda)$ is a cyclic module over the algebra $S_{\mathbb{Z}}^{\cdot}\left(\mathfrak{n}^{-, a}\right)$ having as a generator the image of a highest weight vector $v_{\lambda} \in V(\lambda)$ in $V_{\mathbb{Z}}^{a}(\lambda)$. Hence the module is isomorphic to $S_{\mathbb{Z}}^{\bullet}\left(\mathfrak{n}^{-, a}\right) / I_{\mathbb{Z}}(\lambda)$, where $I_{\mathbb{Z}}(\lambda)$ is the annihilator of $v_{\lambda}$ in $S_{\mathbb{Z}}^{\bullet}\left(\mathfrak{n}^{-, a}\right)$.

We have an additional Lie algebra acting on $S_{\mathbb{Z}}^{\bullet}\left(\mathfrak{n}^{-, a}\right)$ as well as on $V_{\mathbb{Z}}^{a}(\lambda)$. Let $\mathfrak{b}$ be the Borel subalgebra of $\mathfrak{g}=\left(\mathfrak{s l}_{n}\right)_{\mathbb{Z}} \otimes \mathbb{C}$ as in Example 1.1, so $\mathfrak{g}=\mathfrak{n}^{-} \oplus \mathfrak{h} \oplus \mathfrak{n}^{+}$. As free $\mathbb{Z}$-modules, $U_{\mathbb{Z}}\left(\mathfrak{n}^{-}\right) \simeq U_{\mathbb{Z}}(\mathfrak{g}) / U_{\mathbb{Z}}^{+}\left(\mathfrak{h}+\mathfrak{n}^{+}\right)$, so that the adjoint action of $U_{\mathbb{Z}}(\mathfrak{b})$ on $U_{\mathbb{Z}}(\mathfrak{g})$ induces the structure of a $U_{\mathbb{Z}}(\mathfrak{b})$-module on $U_{\mathbb{Z}}\left(\mathfrak{n}^{-}\right)$and hence on $S_{\mathbb{Z}}^{\bullet}\left(\mathfrak{n}^{-, a}\right)$. This action is compatible with the induced $U_{\mathbb{Z}}(\mathfrak{b})$-action on $V_{\mathbb{Z}}^{a}(\lambda)$ [Feigin et al. 2013, Prop. 2.3.]. Recall that for a positive root $\alpha$ we have denoted by $f_{\alpha}$ the corresponding fixed Chevalley basis element in $\left(\mathfrak{s l}_{n}\right)_{-\alpha, \mathbb{Z}}$. Using the presentation of Demazure modules in terms of generators and relations by Joseph, Mathieu and Polo (compare [Mathieu 1989, Lemme 26]), we get as a consequence 
of the proof of Theorem 4.1 the following description of the ideal $I_{\mathbb{Z}}(\lambda)$; see [Feigin et al. 2011a; 2013].

Corollary 4.3. As a cyclic $S_{\mathbb{Z}}\left(\mathfrak{n}^{-, a}\right)$-module, the abelianized module $V_{\mathbb{Z}}^{a}(\lambda)$ is isomorphic to $S_{\mathbb{Z}}^{\bullet}\left(\mathfrak{n}^{-, a}\right) / I_{\mathbb{Z}}(\lambda)$, where

$$
I_{\mathbb{Z}}(\lambda)=S_{\mathbb{Z}}^{\bullet}\left(\mathfrak{n}^{-, a}\right)\left(U_{\mathbb{Z}}\left(\mathfrak{n}^{+}\right) \circ \operatorname{span}\left\{f_{\alpha}^{\left(\left\langle\alpha^{\vee}, \lambda\right\rangle+m\right)} \mid m \geq 1, \alpha>0\right\}\right) \subseteq S_{\mathbb{Z}}\left(\mathfrak{n}^{-, a}\right) .
$$

4C. The proof of Theorem 4.1 will be given in Section 4G, but it needs some preparation. The strategy of the proof is summarized by the following diagram of $S_{\mathbb{Z}}^{\bullet}\left(\mathfrak{n}^{-, a}\right)$-modules. For a dominant weight $\lambda=a_{1} \omega_{1}+\cdots+a_{n-1} \omega_{n-1}$ (so $\lambda^{*}=a_{n-1} \omega_{1}+\cdots+a_{1} \omega_{n-1}$ ), we get the following natural maps (the details are described below):

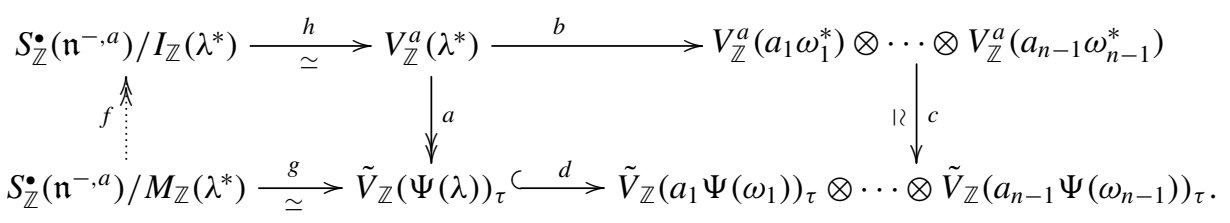

Let us describe the diagram above and the strategy of the proof. We recall that, given a tensor product of cyclic $S_{\mathbb{Z}}^{\bullet}\left(\mathfrak{n}^{-, a}\right)$-modules, the Cartan component of the tensor product is, by definition, the cyclic $S_{\mathbb{Z}}^{\bullet}\left(\mathfrak{n}^{-, a}\right)$-submodule generated by the tensor product of the cyclic generators. Further, recall that the isomorphism $V_{\mathbb{Z}}^{a}\left(\ell \omega_{j}^{*}\right) \simeq$ $\tilde{V}_{\mathbb{Z}}\left(\ell \Psi\left(\omega_{j}\right)\right)_{\tau}$ sends the highest weight vector $v_{\ell \omega_{j}^{*}}$ to the extremal weight vector $v_{\tau\left(\ell \Psi\left(\omega_{j}\right)\right)}$ and uses the Chevalley involution. The maps above are defined as follows:

- $b$ is induced by the compatibility of the PBW filtration with the tensor product, and it is surjective onto the Cartan component of this tensor product.

- $I_{\mathbb{Z}}\left(\lambda^{*}\right)$ is the annihilator in $S_{\mathbb{Z}}^{\bullet}\left(\mathfrak{n}^{-, a}\right)$ of the image of the highest weight vector $v_{\lambda^{*}}$ in $V_{\mathbb{Z}}^{a}\left(\lambda^{*}\right)$ and $h$ is the corresponding quotient map.

- $c$ is the isomorphism given by Proposition 3.4.

- $d$ is the isomorphism onto the Cartan component of the tensor product. The fact that this is an isomorphism follows by standard monomial theory [Lakshmibai et al. 1979] or Frobenius splitting [Ramanathan 1987].

- $a$ equals $c \circ b$ after identifying $\tilde{V}_{\mathbb{Z}}(\Psi(\lambda))_{\tau}$ with its image under $d$.

- $M_{\mathbb{Z}}\left(\lambda^{*}\right)$ is the annihilator in $S_{\mathbb{Z}}^{\bullet}\left(\mathfrak{n}^{-, a}\right)$ of the extremal weight vector $v_{\tau(\Psi(\lambda))}$ in $\tilde{V}_{\mathbb{Z}}(\Psi(\lambda))_{\tau}$ and $g$ is the corresponding quotient map.

- $f$ is going to be constructed in the proof.

In order to finish the proof we will show that $M_{\mathbb{Z}}\left(\lambda^{*}\right) \subseteq I_{\mathbb{Z}}\left(\lambda^{*}\right)$, and the inclusion induces the surjective map $f$ which in turn shows that the map $a$ is an isomorphism. 
4D. We first determine $M_{\mathbb{Z}}\left(\lambda^{*}\right)$. By [Mathieu 1989, Lemme 26], the Demazure module $\tilde{V}_{\mathbb{Z}}(\Psi(\lambda))_{\tau}$ is isomorphic to the algebra $U_{\mathbb{Z}}\left(\tilde{\mathfrak{n}}^{+}\right)$modulo the left ideal $\tilde{I}_{\mathbb{Z}}(\tau \Psi(\lambda))$ generated for all $m \geq 1$ by

$$
\begin{cases}E_{\tilde{\alpha}}^{(m)} & \text { if }\left\langle\tilde{\alpha}^{\vee}, \tau \Psi(\lambda)\right\rangle \geq 0, \\ E_{\tilde{\alpha}}^{\left(-\left\langle\tilde{\alpha}^{\vee}, \tau \Psi(\lambda)\right\rangle+m\right)} & \text { otherwise. }\end{cases}
$$

4E. The annihilator $M_{\mathbb{Z}}\left(\lambda^{*}\right)$ is the intersection of $U_{\mathbb{Z}}\left(\mathfrak{n}^{-, a}\right) \subset U_{\mathbb{Z}}\left(\tilde{\mathfrak{n}}^{+}\right)$with the ideal $\tilde{I}_{\mathbb{Z}}(\tau \Psi(\lambda))$. To determine the intersection, let us divide the positive roots of $\mathrm{SL}_{2 n}$ into three families:

- $\tilde{\alpha}$ is of the first type if $\tilde{\alpha}=\phi(\alpha)$ for some positive $\mathrm{SL}_{n}$-root $\alpha$.

- $\tilde{\alpha}=\tilde{\epsilon}_{k}-\tilde{\epsilon}_{l}$ is of second type if $1 \leq k<l \leq n$ or $n+1 \leq k<l \leq 2 n$.

- $\tilde{\alpha}=\tilde{\epsilon}_{k}-\tilde{\epsilon}_{l}$ is of third type if $1 \leq k \leq n, n+1 \leq l \leq 2 n$ and $k<l-n$.

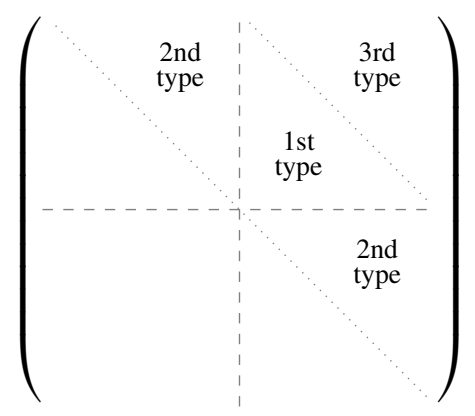

The $E_{\tilde{\alpha}}$, with $\tilde{\alpha}$ of second type, span a Lie subalgebra isomorphic to two copies of $\mathfrak{b}_{\mathbb{Z}}$. Let $\mathfrak{b}_{\mathbb{Z}}^{1}$ denote the first copy spanned by the $E_{\tilde{\alpha}}, \tilde{\alpha}=\tilde{\epsilon}_{k}-\tilde{\epsilon}_{l}, 1 \leq k<l \leq n$, and let $\mathfrak{b}_{\mathbb{Z}}^{2}$ denote the second copy spanned by the $E_{\tilde{\alpha}}, \tilde{\alpha}=\tilde{\epsilon}_{k}-\tilde{\epsilon}_{l}, n+1 \leq k<l \leq 2 n$.

Let $\tilde{I}_{\mathbb{Z}}(\infty) \subset U_{\mathbb{Z}}\left(\tilde{\mathfrak{n}}^{+}\right)$be the left $U_{\mathbb{Z}}\left(\tilde{\mathfrak{n}}^{+}\right)$-submodule generated by the $E_{\tilde{\alpha}}^{(m)}$, with $m \geq 1$ and $\tilde{\alpha}$ of second or third type. Then Lemma 2.5 and a PBW basis argument show that we have the $\mathbb{Z}$-module decomposition

$$
U_{\mathbb{Z}}\left(\tilde{\mathfrak{n}}^{+}\right)=U_{\mathbb{Z}}\left(\mathfrak{n}^{-, a}\right) \oplus \tilde{I}_{\mathbb{Z}}(\infty)=S_{\mathbb{Z}}^{\bullet}\left(\mathfrak{n}^{-, a}\right) \oplus \tilde{I}_{\mathbb{Z}}(\infty) \quad \text { and } \quad \tilde{I}_{\mathbb{Z}}(\infty) \subset \tilde{I}_{\mathbb{Z}}(\tau \Psi(\lambda)) .
$$

By abuse of notation, we identify in the following $S_{\mathbb{Z}}^{\bullet}\left(\mathfrak{n}^{-, a}\right)$ with $U_{\mathbb{Z}}\left(\tilde{\mathfrak{n}}^{+}\right) / \tilde{I}_{\mathbb{Z}}(\infty)$. So determining $M_{\mathbb{Z}}\left(\lambda^{*}\right)=U_{\mathbb{Z}}\left(\mathfrak{n}^{-, a}\right) \cap \tilde{I}_{\mathbb{Z}}(\tau \Psi(\lambda))$ (the intersection taking place in $\left.U_{\mathbb{Z}}\left(\tilde{\mathfrak{n}}^{+}\right)\right)$is equivalent to determining the image of $\tilde{I}_{\mathbb{Z}}(\tau \Psi(\lambda)) / I_{\mathbb{Z}}(\infty)$ in $S_{\mathbb{Z}}^{\bullet}\left(\mathfrak{n}^{-, a}\right)$. In the following we identify $M_{\mathbb{Z}}\left(\lambda^{*}\right)$ with $\tilde{I}_{\mathbb{Z}}(\tau \Psi(\lambda)) / I_{\mathbb{Z}}(\infty)$.

Note that $U_{\mathbb{Z}}\left(\mathfrak{b}_{\mathbb{Z}}^{1} \oplus \mathfrak{b}_{\mathbb{Z}}^{2}\right)$ acts naturally via the adjoint action on $\tilde{\mathfrak{n}}_{\mathbb{Z}}^{+}$and hence on $U_{\mathbb{Z}}\left(\tilde{\mathfrak{n}}^{+}\right)$. The span of the $E_{\tilde{\alpha}}$, with $\tilde{\alpha}$ of second or third type, is stable under this adjoint action of $\mathfrak{b}_{\mathbb{Z}}^{1} \oplus \mathfrak{b}_{\mathbb{Z}}^{2}$, so $\tilde{I}_{\mathbb{Z}}(\infty) \subset U_{\mathbb{Z}}\left(\tilde{\mathfrak{n}}^{+}\right)_{\mathbb{Z}}$ is a submodule with respect to this adjoint action. 
We get an induced $U_{\mathbb{Z}}\left(\mathfrak{b}_{\mathbb{Z}}^{1} \oplus \mathfrak{b}_{\mathbb{Z}}^{2}\right)$-action on $S_{\mathbb{Z}}^{\bullet}\left(\mathfrak{n}^{-, a}\right)$ which we denote by “o". Moreover, since $U_{\mathbb{Z}}^{+}\left(\mathfrak{b}_{\mathbb{Z}}^{1} \oplus \mathfrak{b}_{\mathbb{Z}}^{2}\right)$ (the set of elements without constant term) is contained in $\tilde{I}_{\mathbb{Z}}(\infty)$, we see that $M_{\mathbb{Z}}\left(\lambda^{*}\right)=\tilde{I}_{\mathbb{Z}}(\tau \Psi(\lambda)) / \tilde{I}_{\mathbb{Z}}(\infty)$ is a $U_{\mathbb{Z}}^{+}\left(\mathfrak{b}_{\mathbb{Z}}^{1} \oplus \mathfrak{b}_{\mathbb{Z}}^{2}\right)$-stable submodule with respect to the "o"-action of $U_{\mathbb{Z}}\left(\mathfrak{b}_{\mathbb{Z}}^{1} \oplus \mathfrak{b}_{\mathbb{Z}}^{2}\right)$. As a first step in the proof of the theorem we show:

Lemma 4.4. The left $S_{\mathbb{Z}}^{\bullet}\left(\mathfrak{n}^{-, a}\right)$-submodule $M_{\mathbb{Z}}\left(\lambda^{*}\right) \subset S_{\mathbb{Z}}^{\bullet}\left(\mathfrak{n}^{-, a}\right)$ is generated by

$$
\left.m_{\mathbb{Z}}\left(\lambda^{*}\right):=\left\langle U_{\mathbb{Z}}\left(\mathfrak{b}_{\mathbb{Z}}^{1} \oplus \mathfrak{b}_{\mathbb{Z}}^{2}\right) \circ E_{\tilde{\alpha}}^{\left(-\left\langle\tilde{\alpha}^{\vee}, \tau \Psi(\lambda)\right\rangle+m\right)}\right| \tilde{\alpha} \text { of first type and } m \geq 1\right\rangle_{\mathbb{Z}} .
$$

Proof. Let $\overline{\mathrm{m}}$ be an element of $M_{\mathbb{Z}}\left(\lambda^{*}\right)$ and choose a representative $\mathrm{m}$ in $\tilde{I}_{\mathbb{Z}}(\tau \Psi(\lambda))$. Since we are free to choose a representative modulo $I_{\mathbb{Z}}(\infty)$, we may assume (see Section 4D) that $\mathrm{m}$ is a sum of monomials of the form $r E_{\tilde{\alpha}}^{(\ell)}$, where $r$ is a monomial in the $E_{\tilde{\beta}}^{(q)}$ with $q \geq 0$ and $\tilde{\beta}$ of first, second, or third type, and $\ell=-\left\langle\tilde{\alpha}^{\vee}, \tau \Psi(\lambda)\right\rangle+k$ for some $k \geq 1$ and $\tilde{\alpha}$ of first type.

If $\tilde{\gamma}$ is a root of third type and $\tilde{\beta}$ is any other positive root, then $\left[E_{\tilde{\gamma}}, E_{\tilde{\beta}}\right]=c E_{\tilde{\gamma}^{\prime}}$, where $c \in \mathbb{Z}$ and either $c=0$ or $\tilde{\gamma}^{\prime}$ is of third type. So if $r$ has a factor $E_{\tilde{\gamma}}^{(p)}$, with $p>0$ and $\tilde{\gamma}$ a root of third type, then we can rewrite the monomial $r E_{\tilde{\alpha}}^{(\ell)}$ as a sum of monomials of the form $r^{\prime} E_{\tilde{\gamma}}^{\left(p^{\prime}\right)}$, with $p^{\prime}>0$. Since this sum is an element in $I_{\mathbb{Z}}(\infty)$, without loss of generality we will assume in the following that $r$ has only factors of the form $E_{\tilde{\beta}}^{(\ell)}$, with $\tilde{\beta}$ of first or second type.

If $\tilde{\gamma}$ is of second type and $\tilde{\beta}$ is of first type, then $\left[E_{\tilde{\gamma}}, E_{\tilde{\beta}}\right]=c E_{\tilde{\gamma}^{\prime}}$, where $c \in \mathbb{Z}$ and either $c=0$ or $\tilde{\gamma}^{\prime}$ is of first or third type. So after reordering the factors we can assume without loss of generality in the following that $r E_{\tilde{\alpha}}^{(\ell)}$ is of the form $r=r_{1} r_{2}$, where $r_{1}$ is a monomial in the $E_{\tilde{\beta}}^{(\ell)}$, with $\tilde{\beta}$ of first type, and $r_{2}$ is a monomial in the $E_{\tilde{\gamma}}^{(\ell)}$, with $\tilde{\gamma}$ of second type.

Recall that for $\tilde{\gamma}$ of second type,

$$
\begin{aligned}
E_{\tilde{\gamma}} E_{\tilde{\beta}_{1}} \cdots E_{\tilde{\beta}_{m}} & \equiv \sum_{i=1}^{m} E_{\tilde{\beta}_{1}} \cdots E_{\tilde{\beta}_{i-1}}\left(E_{\tilde{\gamma}} E_{\tilde{\beta}_{i}}-E_{\tilde{\beta}_{i}} E_{\tilde{\gamma}}\right) E_{\tilde{\beta}_{i+1}} \cdots E_{\tilde{\beta}_{m}} \bmod I_{\mathbb{Z}}(\infty) \\
& \equiv \sum_{i=1}^{m} E_{\tilde{\beta}_{1}} \cdots E_{\tilde{\beta}_{i-1}}\left(\operatorname{ad}\left(E_{\tilde{\gamma}}\right)\left(E_{\tilde{\beta}_{i}}\right)\right) E_{\tilde{\beta}_{i+1}} \cdots E_{\tilde{\beta}_{m}} \bmod I_{\mathbb{Z}}(\infty) \\
& \equiv \operatorname{ad}\left(E_{\tilde{\gamma}}\right)\left(E_{\tilde{\beta}_{1}} \cdots E_{\tilde{\beta}_{m}}\right) \bmod I_{\mathbb{Z}}(\infty) .
\end{aligned}
$$

An appropriate reformulation of the equality above holds also for the divided powers of root vectors. It follows that $r_{2} E_{\tilde{\alpha}}^{(\ell)} \in m_{\mathbb{Z}}\left(\lambda^{*}\right)$; hence $r E_{\tilde{\alpha}}^{(\ell)}=r_{1} r_{2} E_{\tilde{\alpha}}^{(\ell)} \in$ $S_{\mathbb{Z}}^{\bullet}\left(\mathfrak{n}^{-, a}\right) m_{\mathbb{Z}}\left(\lambda^{*}\right)$, which implies that $M_{\mathbb{Z}}\left(\lambda^{*}\right)$ is generated by $m_{\mathbb{Z}}\left(\lambda^{*}\right)$ as a left $S_{\mathbb{Z}}^{\bullet}\left(\mathfrak{n}^{-, a}\right)$-module.

4F. To compare $M_{\mathbb{Z}}\left(\lambda^{*}\right)$ with $I_{\mathbb{Z}}\left(\lambda^{*}\right)$, we need a variant of the description of $m_{\mathbb{Z}}\left(\lambda^{*}\right)$. Let $\Delta\left(\mathfrak{b}_{\mathbb{Z}}\right) \subset \mathfrak{b}_{\mathbb{Z}}^{1} \oplus \mathfrak{b}_{\mathbb{Z}}^{2}$ be the Lie subalgebra obtained as a diagonally embedded copy of $\mathfrak{b}_{\mathbb{Z}}$. Let $U_{\mathbb{Z}}\left(\Delta\left(\mathfrak{b}_{\mathbb{Z}}\right)\right) \subset U_{\mathbb{Z}}\left(\left(\mathfrak{b}_{\mathbb{Z}}^{1}\right) \oplus\left(\mathfrak{b}_{\mathbb{Z}}^{2}\right)\right)$ be its hyperalgebra. 
Lemma 4.5. $m_{\mathbb{Z}}\left(\lambda^{*}\right)=\left\langle U_{\mathbb{Z}}\left(\Delta\left(\mathfrak{b}_{\mathbb{Z}}\right)\right) \circ E_{\tilde{\alpha}}^{\left(-\left\langle\tilde{\alpha}^{\vee}, \tau \Psi(\lambda)\right\rangle+m\right)}\right| \tilde{\alpha}$ of first type and $\left.m \geq 1\right\rangle_{\mathbb{Z}}$.

Proof. We assume first that $k$ is an algebraically closed field of arbitrary characteristic. Let $B$ be the subgroup of upper triangular invertible matrices in $\mathrm{SL}_{n}(k)$, so Lie $B=\mathfrak{b}$. Let $B^{1} \times B^{2} \subset \mathrm{SL}_{2 n}(k)$ be the subgroup with Lie algebra $\mathfrak{b}^{1} \oplus \mathfrak{b}^{2}$ and denote by $\Delta(B) \subset B^{1} \times B^{2}$ the diagonally embedded group isomorphic to $B$.

Let $\mathfrak{q}$ be the sum of the $\mathrm{SL}_{2 n}$-root spaces corresponding to roots of second or third type. Then $\tilde{\mathfrak{n}}^{+}=\mathfrak{n}^{-, a} \oplus \mathfrak{q}$ and we identify $\mathfrak{n}^{-, a}$ with $\tilde{\mathfrak{n}}^{+} / \mathfrak{q}$. The adjoint action of $B^{1} \times B^{2}$ on $\mathfrak{s l}_{2 n}$ admits $\tilde{\mathfrak{n}}^{+}$as well as $\mathfrak{q}$ as submodules, so we get an induced $\left(B^{1} \times B^{2}\right)$-action on $\mathfrak{n}^{-, a}=\tilde{\mathfrak{n}}^{+} / \mathfrak{q}$. This action naturally extends to the commutative hyperalgebra $S_{k}^{\bullet}\left(\mathfrak{n}^{-, a}\right)$.

If we replace the group action of $B^{1} \times B^{2}$ by the induced action of the hyperalgebra $U_{k}\left(\mathfrak{b}^{1} \oplus \mathfrak{b}^{2}\right)$ of the group, then we get the action of $U_{k}\left(\mathfrak{b}^{1} \oplus \mathfrak{b}^{2}\right)$ on $U_{k}\left(\tilde{\mathfrak{n}}^{+}\right)$, respectively on $S_{k}^{\bullet}\left(\mathfrak{n}^{-, a}\right)$ discussed above, and similarly for the action of $\Delta(B)$ and its hyperalgebra $U_{k}(\Delta(\mathfrak{b}))$. Recall that for a root $\tilde{\alpha}$ of type 1 ,

$$
U_{k}\left(\mathfrak{b}^{1} \oplus \mathfrak{b}^{2}\right) \circ E_{\tilde{\alpha}}^{(m)}=\left\langle\operatorname{Ad}\left(\left(b^{1}, b^{2}\right)\right) \circ\left(E_{\tilde{\alpha}}\right)^{(m)} \mid\left(b_{1}, b_{2}\right) \in B^{1} \times B^{2}\right\rangle,
$$

i.e., the smallest $U_{k}\left(\mathfrak{b}^{1} \oplus \mathfrak{b}^{2}\right)$ stable subspace containing $\left(E_{\tilde{\alpha}}\right)^{(m)}$ is the linear span of the $\left(B^{1} \times B^{2}\right)$-orbit. The same holds in the other case, so we have:

$$
U_{k}(\Delta(\mathfrak{b})) \circ E_{\tilde{\alpha}}^{(m)}=\left\langle\operatorname{Ad}((b, b)) \circ\left(E_{\tilde{\alpha}}\right)^{(m)} \mid b \in B\right\rangle .
$$

Let $\mathfrak{d}$ be the sum of the $\mathrm{SL}_{2 n}$-root spaces corresponding to roots of first or third type and let $\mathfrak{d}^{3}$ be just the sum of the root spaces corresponding to roots of third type, so $\mathfrak{d}=\mathfrak{n}^{-, a} \oplus \mathfrak{d}^{3}$. We identify $\mathfrak{d} \subset \mathfrak{s l}_{2 n}$ with $M_{n}(k)$, formally this can be done by the map

$$
\chi: \mathfrak{d} \rightarrow M_{n}(k), \quad \tilde{A}=\left(\begin{array}{cc}
0 & A \\
0 & 0
\end{array}\right) \mapsto A,
$$

where $A$ is a $n \times n$ matrix. In the following we simplify the notation and omit the map $\chi$. We freely identify $\mathfrak{d}$ with $M_{n}(k)$, so we denote by $A$ the $n \times n$ matrix as well as the $2 n \times 2 n$-matrix $\tilde{A} \in \mathfrak{d}$. Note that for $\left(b_{1}, b_{2}\right) \in B^{1} \times B^{2}$ we get

$$
\operatorname{Ad}\left(\left(b_{1}, b_{2}\right)\right) \circ \tilde{A}=\left(\begin{array}{cc}
b_{1} & 0 \\
0 & b_{2}
\end{array}\right)\left(\begin{array}{cc}
0 & A \\
0 & 0
\end{array}\right)\left(\begin{array}{cc}
b_{1}^{-1} & 0 \\
0 & b_{2}^{-1}
\end{array}\right)=\left(\begin{array}{cc}
0 & b_{1} A b_{2}^{-1} \\
0 & 0
\end{array}\right),
$$

we just write $\operatorname{Ad}\left(\left(b_{1}, b_{2}\right)\right) \circ(A)=b_{1} A b_{2}^{-1}$ and $\operatorname{Ad}((b, b)) \circ(A)=b A b^{-1}$. Recall that $\chi$ is just a vector space isomorphism. If we equip in addition $M_{n}(k)$ with the trivial Lie bracket, then this becomes also a Lie algebra homomorphism. In this sense we identify also the (commutative) Lie subalgebras $\mathfrak{n}^{-, a}$ and $\mathfrak{d}^{3}$ with subalgebras of $M_{n}$. An elementary calculation shows how the $B^{1} \times B^{2}$-orbit through $E_{\tilde{\alpha}}=E_{\epsilon_{n}-\epsilon_{n+1}}$ breaks up into $\Delta(B)$-orbits. Recall that we identify $\mathfrak{d}$ with $M_{n}(k)$ 
and $E_{\epsilon_{n}-\epsilon_{n+1}} \in \mathfrak{d}$ corresponds to $E_{n, 1}$ :

$$
\begin{aligned}
\left\{\operatorname{Ad}\left(\left(b_{1}, b_{2}\right)\right) \circ\left(E_{\tilde{\alpha}}\right) \mid\left(b_{1}, b_{2}\right) \in B^{1} \times B^{2}\right\} & =\left\{b_{1} E_{n, 1} b_{2}^{-1} \mid b_{1}, b_{2} \in B\right\} \\
& =\bigcup_{\lambda \in k}\left\{b\left(E_{n, 1}+\lambda E_{1,1}\right) b^{-1} \mid b \in B\right\} \\
& \subset M_{n}(k) .
\end{aligned}
$$

We conclude for the linear span,

$$
\begin{aligned}
U_{k}\left(\mathfrak{b}^{1} \oplus \mathfrak{b}^{2}\right) \circ E_{\tilde{\alpha}}^{(m)} & =\left\langle\operatorname{Ad}\left(\left(b_{1}, b_{2}\right)\right) \circ\left(E_{\tilde{\alpha}}\right)^{(m)} \mid\left(b_{1}, b_{2}\right) \in B^{1} \times B^{2}\right\rangle \\
& =\left\langle\left(\operatorname{Ad}\left(\left(b_{1}, b_{2}\right)\right) \circ\left(E_{\tilde{\alpha}}\right)\right)^{(m)} \mid\left(b_{1}, b_{2}\right) \in B^{1} \times B^{2}\right\rangle \\
& =\left\langle\left(b_{1} E_{n, 1} b_{2}^{-1}\right)^{(m)} \mid b_{1}, b_{2} \in B\right\rangle \\
& =\left\langle\bigcup_{\lambda \in k}\left\{b\left(E_{n, 1}+\lambda E_{1,1}\right)^{(m)} b^{-1} \mid b \in B\right\}\right\rangle .
\end{aligned}
$$

Let $I\left(\mathfrak{d}^{3}\right) \subset U_{k}(\mathfrak{d})$ be the left ideal in the hyperalgebra generated by $\mathfrak{d}^{3}$. Then

$$
\begin{aligned}
U_{k}\left(\mathfrak{b}^{1} \oplus \mathfrak{b}^{2}\right) \circ E_{\tilde{\alpha}}^{(m)} & \equiv\left\langle\bigcup_{\lambda \in k}\left\{\left(b\left(E_{n, 1}+\lambda E_{1,1}\right) b^{-1}\right)^{(m)} \mid b \in B\right\}\right\rangle \\
& \equiv\left\langle\left(b E_{n, 1} b^{-1}\right)^{(m)} \mid b \in B\right\rangle \bmod I\left(\mathfrak{d}^{3}\right)
\end{aligned}
$$

because the $E_{1,1}^{(\ell)}$, with $\ell \geq 1$, lie in the $\Delta(B)$-stable ideal $I\left(\mathfrak{d}^{3}\right)$. It follows that

$$
\begin{aligned}
U_{k}\left(\mathfrak{b}^{1} \oplus \mathfrak{b}^{2}\right) \circ E_{\tilde{\alpha}}^{(m)} & \equiv\left\langle\operatorname { A d } ( ( b , b ) ) \left( E_{\tilde{\alpha}}^{(m)}|b \in B\rangle\right.\right. \\
& \equiv U_{k}(\Delta(\mathfrak{b})) \circ E_{\tilde{\alpha}}^{(m)} \bmod I\left(\mathfrak{d}^{3}\right) .
\end{aligned}
$$

Since $I\left(\mathfrak{d}^{3}\right) \subset \tilde{I}_{k}(\infty)$, the equation holds in $S^{\bullet}\left(\mathfrak{n}^{-, a}\right)=U_{k}\left(\tilde{\mathfrak{n}}^{+}\right) / \tilde{I}(\infty)$ too, so

$$
U_{k}\left(\mathfrak{b}^{1} \oplus \mathfrak{b}^{2}\right) \circ E_{\tilde{\alpha}}^{(m)}=U_{k}(\Delta(\mathfrak{b})) \circ E_{\tilde{\alpha}}^{(m)}
$$

in $S^{\bullet}\left(\mathfrak{n}^{-, a}\right)$. It is now easy to see that the same arguments prove the equality for all $E_{\tilde{\alpha}}^{(m)}$, with $\tilde{\alpha}$ of first type and $m \geq 1$. Clearly,

$$
U_{\mathbb{Z}}\left(\mathfrak{b}^{1} \oplus \mathfrak{b}^{2}\right) \circ E_{\tilde{\alpha}}^{(m)} \supseteq U_{\mathbb{Z}}(\Delta(\mathfrak{b})) \circ E_{\tilde{\alpha}}^{(m)} .
$$

Since we have equality after base change for fields of arbitrary characteristics, the equality of the modules holds also over $\mathbb{Z}$. In particular, the following equality holds in $S_{\mathbb{Z}}^{\bullet}\left(\mathfrak{n}^{-, a}\right)$ :

$$
\begin{aligned}
m_{\mathbb{Z}}\left(\lambda^{*}\right) & \left.=\left\langle U_{\mathbb{Z}}\left(\mathfrak{b}_{\mathbb{Z}}^{1} \oplus \mathfrak{b}_{\mathbb{Z}}^{2}\right) \circ E_{\tilde{\alpha}}^{\left(-\left\langle\tilde{\alpha}^{\vee}, \tau \Psi(\lambda)\right\rangle+m\right)}\right| \tilde{\alpha} \text { of first type and } m \geq 1\right\rangle_{\mathbb{Z}} \\
& \left.=\left\langle U_{\mathbb{Z}}\left(\Delta\left(\mathfrak{b}_{\mathbb{Z}}\right)\right) \circ E_{\tilde{\alpha}}^{\left(-\left\langle\tilde{\alpha}^{\vee}, \tau \Psi(\lambda)\right\rangle+m\right)}\right| \tilde{\alpha} \text { of first type and } m \geq 1\right\rangle_{\mathbb{Z}} .
\end{aligned}
$$


4G. Proof of Theorem 4.1. Recall the identification of the abelianized version of $\mathfrak{n}^{-} \subset \mathfrak{s l}_{n}$ with $\mathfrak{n}^{-, a} \subset \mathfrak{s l}_{2 n}$, which sends the image of a Chevalley generator $f_{\alpha}$ to $E_{\phi(\alpha)}$. By Lemma 2.5 (see Lemma 3.3 for the twist $\lambda \leftrightarrow \lambda^{*}$ ) the elements $E_{\tilde{\alpha}}^{\left(-\left\langle\tilde{\alpha}^{\vee}, \tau \Psi(\lambda)\right\rangle+m\right)}$, where $\tilde{\alpha}$ is of first type and $m \geq 1$ are elements of $I_{\mathbb{Z}}\left(\lambda^{*}\right)$. Now the $U_{\mathbb{Z}}(\mathfrak{b})$-module structure on $S_{\mathbb{Z}}^{\bullet}\left(\mathfrak{n}^{-, a}\right)$ described in Section 4B is the same as the one described above, so it follows that $m_{\mathbb{Z}}\left(\lambda^{*}\right) \subset I_{\mathbb{Z}}\left(\lambda^{*}\right)$ and hence $M_{\mathbb{Z}}\left(\lambda^{*}\right) \subset I_{\mathbb{Z}}\left(\lambda^{*}\right)$, which, as explained in Section 4C, finishes the proof of the theorem.

4H. Let $\rho$ be the sum of all fundamental weights for $\mathrm{SL}_{n}$ and set $\tilde{\rho}=\Psi(\rho)$. Let $Q_{\mathbb{Z}} \subset\left(\mathrm{SL}_{2 n}\right)_{\mathbb{Z}}$ be the corresponding parabolic $\mathbb{Z}$-subgroup. Recall that $\left(N^{-, a}\right)_{\mathbb{Z}}$ is a commutative subgroup of the Borel subgroup $\tilde{B}_{\mathbb{Z}}$. For any $\mathrm{SL}_{2 n}$-root $\tilde{\alpha}$ let $U_{\mathbb{Z}, \tilde{\alpha}}$ be the associated root subgroup.

Lemma 4.6. The orbit $\tilde{B}_{\mathbb{Z}} \cdot \tau \subset\left(\mathrm{SL}_{2 n}\right)_{\mathbb{Z}} / Q_{\mathbb{Z}}$ is equal to $N^{-, a} \cdot \tau$, and the map $N^{-, a} \rightarrow N^{-, a} \cdot \tau, u \mapsto u \tau$, is a bijection.

Proof. We have $\tilde{B}_{\mathbb{Z}} \cdot \tau=\prod_{\tilde{\alpha}>0} U_{\mathbb{Z}, \tilde{\alpha}} \cdot \tau$, and the map $\prod_{\tilde{\alpha} \in \Gamma} U_{\mathbb{Z}, \tilde{\alpha}} \rightarrow \prod_{\tilde{\alpha} \in \Gamma} U_{\mathbb{Z}, \tilde{\alpha}} \cdot \tau$ is a bijection, where $\Gamma$ is the set of all positive roots of $\operatorname{SL}_{2 n}$ such that $\tau^{-1}(\tilde{\alpha})<0$ and $\tau^{-1}(\tilde{\alpha})$ is not an element of the root system of $Q_{\mathbb{Z}}$. Now this condition is fulfilled if and only if $\left\langle\tau^{-1}\left(\tilde{\alpha}^{\vee}\right), \tilde{\rho}\right\rangle<0$, or, equivalently, $\left\langle\tilde{\alpha}^{\vee}, \tau(\tilde{\rho})\right\rangle<0$. By Lemma 2.4 this is only possible if $\tilde{\alpha}=\tilde{\epsilon}_{i}-\tilde{\epsilon}_{j}$ is such that $1 \leq i \leq n, n+1 \leq j \leq 2 n$, and $i>j-n$. But this implies that the root subgroup $U_{\mathbb{Z}, \tilde{\alpha}}$ is a subgroup of $N^{-, a}$, and all root subgroups of $\left(\mathrm{SL}_{2 n}\right)_{\mathbb{Z}}$ contained in $N^{-, a}$ satisfy this condition. It follows that $N^{-, a}$ is the product of all root subgroups corresponding to positive roots of $\mathrm{SL}_{2 n}$ in $\Gamma$.

Recall that the degenerate flag scheme $\mathcal{F} \ell(\lambda)_{\mathbb{Z}}^{a}$ is the closure of the $N_{\mathbb{Z}}^{-, a}$-orbit through the highest weight vector in $\mathbb{P} P\left(V_{\mathbb{Z}}^{a}(\lambda)\right)$.

Theorem 4.7. Let $\lambda$ be a dominant weight for $\mathrm{SL}_{n}$. The Schubert scheme $X_{\mathbb{Z}}(\tau) \subset$ $\mathbb{P}\left(\tilde{V}_{\mathbb{Z}}(\Psi(\lambda))_{\tau}\right)$ is isomorphic to the degenerate partial flag scheme $\mathcal{F} \ell\left(\lambda^{*}\right)_{\mathbb{Z}}^{a}$ for $\left(\mathrm{SL}_{n}\right)_{\mathbb{Z}}$, and this map induces a module isomorphism $H^{0}\left(X_{\mathbb{Z}}(\tau), \mathcal{L}_{\Psi(\lambda)}\right) \simeq\left(V_{\mathbb{Z}}^{a}\left(\lambda^{*}\right)\right)^{*}$.

Proof. We consider only the case where $\lambda$ is regular; the arguments in the general case are similar. With respect to the isomorphism in Lemma 4.6, the orbit

$$
\tilde{B}_{\mathbb{Z}} \cdot \tau \subset\left(\mathrm{SL}_{2 n}\right)_{\mathbb{Z}} /\left(\tilde{P}_{\lambda}\right)_{\mathbb{Z}} \hookrightarrow \mathbb{P}(\tilde{V}(\Psi(\lambda))),
$$

through the extremal weight vector, which is the same as the $N^{-, a}$-orbit, is mapped onto the $N_{\mathbb{Z}}^{-, a}$-orbit through the highest weight vector in $\mathbb{P}\left(V_{\mathbb{Z}}^{a}\left(\lambda^{*}\right)\right)$. By definition, the Schubert scheme $X_{\mathbb{Z}}(\tau)$ is the closure of the orbit $\tilde{B}_{\mathbb{Z}} \cdot \tau$ and the degenerate flag scheme $\mathcal{F} \ell\left(\lambda^{*}\right)_{\mathbb{Z}}^{a}$ is the closure of the $N_{\mathbb{Z}}^{-, a}$-orbit. It follows that the module isomorphism induces an isomorphism between the Schubert scheme $X_{\mathbb{Z}}(\tau) \subseteq$ $\mathbb{P}\left(\tilde{V}_{\mathbb{Z}}(\Psi(\lambda))_{\tau}\right)$ and the degenerate flag scheme $\mathcal{F} \ell\left(\lambda^{*}\right)_{\mathbb{Z}}^{a}$ in $\mathbb{P}\left(V_{\mathbb{Z}}^{a}(\lambda)\right)$. Hence we get induced isomorphisms

$$
H^{0}\left(X_{\mathbb{Z}}(\tau), \mathcal{L}_{\Psi(\lambda)}\right) \simeq\left(\tilde{V}_{\mathbb{Z}}(\Psi(\lambda))_{\tau}\right)^{*} \simeq\left(V_{\mathbb{Z}}^{a}\left(\lambda^{*}\right)\right)^{*}
$$


for the dual modules.

Let $k$ be an algebraically closed field of arbitrary characteristic and denote by $V_{k}(\lambda)=V_{\mathbb{Z}}(\lambda) \otimes_{\mathbb{Z}} k, U_{k}\left(\mathfrak{s l}_{n}\right)=U_{\mathbb{Z}}\left(\mathfrak{s l}_{n}\right) \otimes_{\mathbb{Z}} k, U_{k}\left(\mathfrak{n}^{-}\right)=U_{\mathbb{Z}}\left(\mathfrak{n}^{-}\right) \otimes_{\mathbb{Z}} k$, etc., the objects obtained by base change. The PBW filtration

$$
V_{k}(\lambda)_{\ell}=\left\langle Y_{1}^{\left(m_{1}\right)} \cdots Y_{N}^{\left(m_{N}\right)} v_{\lambda} \mid m_{1}+\cdots+m_{N} \leq \ell, Y_{1}, \ldots, Y_{N} \in \mathfrak{n}_{k}^{-}\right\rangle
$$

and the associated graded space $V_{k}^{a}(\lambda)$ is defined in the same way as before, and by Corollary 4.2, $V_{k}(\lambda)_{\ell}=V_{\mathbb{Z}}(\lambda)_{\ell} \otimes_{\mathbb{Z}} k$ and $V_{k}^{a}(\lambda)=V_{\mathbb{Z}}^{a}(\lambda) \otimes_{\mathbb{Z}} k$. The group $N_{k}^{-, a}$ acts on the abelianized representation $V_{k}^{a}(\lambda)$, and the degenerate flag variety $\mathcal{F} \ell(\lambda)_{k}^{a}$ is the closure of the $N_{k}^{-, a}$-orbit through the highest weight vector in $\mathbb{P}\left(V_{k}^{a}(\lambda)\right)$.

Now by the results of [Mathieu 1989; Mehta and Ramanathan 1988; Ramanathan 1987; Ramanan and Ramanathan 1985] one knows that for Demazure modules we have $\tilde{V}_{k}(\Psi(\lambda))_{\tau}=\tilde{V}_{\mathbb{Z}}(\Psi(\lambda))_{\tau} \otimes_{\mathbb{Z}} k, X_{k}(\tau)=X_{\mathbb{Z}}(\tau) \otimes_{\mathbb{Z}} k$, etc., and that the Schubert varieties are Frobenius split, projectively normal and have rational singularities. It follows that $V_{k}^{a}\left(\lambda^{*}\right)=\tilde{V}_{k}(\Psi(\lambda))_{\tau}$ and $\mathcal{F} \ell\left(\lambda^{*}\right)_{k}^{a}=X_{k}(\tau)$, so the degenerate flag variety has in this case the same nice geometric properties as the Schubert variety. For a dominant $\mathrm{SL}_{n}$-weight $\lambda=\sum_{i=1}^{n-1} a_{i} \omega_{i}$, let the support $\operatorname{supp} \lambda$ of $\lambda$ be the set $\left\{i \mid 1 \leq i \leq n-1, a_{i} \neq 0\right\}$.

Corollary 4.8. The degenerate partial flag variety $\mathcal{F} \ell(\lambda)_{k}^{a}$ depends only on supp $\lambda$. It is a projectively normal variety, Frobenius split, with rational singularities.

Remark 4.9. Feigin and Finkelberg [2013] construct resolutions of the degenerate flag varieties given by towers of $\mathbb{P}^{1}$-fibrations. The steps of the successive fibrations are indexed by the set of positive roots, which had been totally reordered. In fact, their varieties are Bott-Samelson varieties [Cerulli Irelli and Lanini 2015, Appendix] and such an order (which actually should be thought of as an order on the set of negative roots) is now natural since it corresponds to the subsequent steps of the Bott-Samelson variety indexed by the reduced expression (2) of $\tau$, under the identification of $-\alpha_{i, j}$ with $\tilde{\alpha}_{j, i+n}$.

\section{A special Schubert variety: the $\mathrm{Sp}_{2 m}$ case}

As for the $\mathrm{SL}_{n}$ case, we want to realize for Example 1.2 the abelianized representation $V_{\mathbb{Z}}(\lambda)^{a}$ for $N_{\mathbb{Z}}^{-, a}$ as a Demazure submodule in an irreducible representation for the larger group $\mathrm{Sp}_{2(2 m)}$.

5A. A special Weyl group element. Let us keep the same notation as in the previous sections and denote by $\mathfrak{h} \subset \mathfrak{s p}_{2 m}$ (resp., $\tilde{\mathfrak{h}} \subset \mathfrak{s p}_{2(2 m)}$ ) the Cartan subalgebra of traceless complex diagonal matrices and by $\mathfrak{b} \subset \mathfrak{s p}_{2 m}$ (resp., $\tilde{\mathfrak{b}} \subset \mathfrak{s p}_{2(2 m)}$ ) the Borel subalgebra of traceless complex upper triangular matrices. Let $\left\{\epsilon_{1}, \ldots, \epsilon_{2 m}\right\}$ (resp., $\left.\left\{\tilde{\epsilon}_{1}, \ldots, \tilde{\epsilon}_{2(2 m)}\right\}\right)$ be a basis of the dual vector space $\mathfrak{h}^{*}\left(\right.$ resp., $\left.\tilde{\mathfrak{h}}^{*}\right)$. The choice of 
Cartan and Borel subalgebras we made determines the following set of positive roots for $\mathrm{Sp}_{2 m}$ :

$$
\alpha_{i, j}:= \begin{cases}\epsilon_{i}-\epsilon_{j} & 1 \leq i<j \leq m, \\ \epsilon_{i}+\epsilon_{j-m} & 1 \leq i \leq m<j \text { and } i+j \leq 2 m,\end{cases}
$$

where the simple roots are $\left\{\alpha_{i}:=\alpha_{i, i+1} \mid 1 \leq i \leq m-1\right\} \cup\left\{\alpha_{m}:=2 \epsilon_{m}\right\}$. We will write $\tilde{\alpha}_{i, j}$ for the $\mathrm{Sp}_{2 m}$-roots. The Weyl group of $\mathrm{Sp}_{2(2 m)}$ is denoted $\widetilde{W}$. This is the group of linear transformations of $\mathfrak{h}^{*}$ generated by the elements $\left\{r_{i} \mid 1 \leq i \leq 2 m\right\}$, where $r_{i}$ denotes the reflection with respect to the simple root $\tilde{\alpha}_{i}$.

Definition 5.1. We define in $\widetilde{W}$ a very special element:

$\bar{\tau}=\left(r_{2 m} \cdots r_{m+1}\right) \cdots$

$$
\left(r_{2 m} r_{2 m-1} r_{2 m-2}\right)\left(r_{2 m} r_{2 m-1}\right) r_{2 m}\left(r_{m} \cdots r_{2 m-2}\right) \cdots\left(r_{4} r_{5} r_{6}\right)\left(r_{3} r_{4}\right) r_{2} .
$$

Any element of the Weyl group $\widetilde{W}$ of $\mathrm{Sp}_{2(2 m)}$ can be identified with an element of the symmetric group on $4 m$ letters $\mathcal{S}_{4 m}$, via $r_{i}=s_{i} s_{4 m-i}$, for $1 \leq i \leq 2 m-1$, and $r_{n}=s_{2 m}$ (where, as usual, $s_{i}$ denotes the transposition exchanging $i$ and $i+1$ ) and it acts on the basis $\left\{e_{i} \mid i=1, \ldots, 4 m\right\}$ of $\mathbb{C}^{4 m}$ by permuting the indices. It is an easy check to see that under this identification $\bar{\tau}$ equals the element $\tau$ of Definition 2.2 for $n=2 m$ and we hence have the following (compare Lemma 2.3):

Lemma 5.2. In the irreducible $\operatorname{Sp}_{2(2 m)}$-representation $\tilde{V}\left(\tilde{\omega}_{2 i}\right) \subset \wedge^{2 i} \mathbb{C}^{4 m}$, with $1 \leq i \leq 2 m$, let $v_{\omega_{2 i}} v_{\omega_{2 i}}=e_{1} \wedge e_{2} \wedge \ldots \wedge e_{2 i}$ be the highest weight vector. Then (up to sign),

$$
\bar{\tau}\left(v_{\omega_{2 i}}\right)=e_{1} \wedge e_{2} \wedge \cdots \wedge e_{i} \wedge e_{2 m+1} \wedge e_{2 m+2} \wedge \cdots \wedge e_{2 m+i} .
$$

We denote by $\left\{\omega_{i} \mid 1 \leq i \leq m\right\}$, resp. $\left\{\tilde{\omega}_{i} \mid 1 \leq i \leq 2 m\right\}$, the fundamental weights of $\mathfrak{s p}_{2 m}$, resp., $\mathfrak{s p}_{2(2 m)}$. They are characterized by the property $\left\langle\alpha_{i}^{\vee}, \omega_{j}\right\rangle=\delta_{i, j}$.

Definition 5.3. Let $\Psi: \mathfrak{h}^{*} \rightarrow \tilde{\mathfrak{h}}^{*}$ be the linear map defined on the weight lattice by

$$
\Psi\left(\sum_{i=1}^{n-1} a_{i} \omega_{i}\right):=\sum_{i=1}^{n-1} a_{i} \tilde{\omega}_{2 i} .
$$

Note: $\Psi$ sends dominant weights to dominant weights. Let $\lambda=b_{1} \epsilon_{1}+\cdots+b_{m} \epsilon_{m}$, with $b_{1} \geq \cdots \geq b_{m} \geq 0$, be a dominant weight for $\mathrm{Sp}_{2 m}$.

Lemma 5.4. $\bar{\tau}(\Psi(\lambda))=b_{1} \tilde{\epsilon}_{1}+\cdots+b_{m} \tilde{\epsilon}_{m}-b_{m} \tilde{\epsilon}_{m+1}-\cdots-b_{1} \tilde{\epsilon}_{2 m}$.

Proof. This equality follows directly from Lemma 5.2 above.

As in the special linear case, we define a map from the set of negative roots of $\mathrm{Sp}_{2 m}$ to the set of positive $\mathrm{Sp}_{2(2 m)}$-roots by sending $\alpha_{i, j}$ to $\tilde{\alpha}_{j, i+2 m}$. The following is the symplectic analogue of Lemma 2.5: 
Lemma 5.5. (i) Let $\lambda$ be a dominant weight for $\mathrm{Sp}_{2 m}(\mathbb{C})$. For a positive $\mathrm{Sp}_{2(2 m)^{-}}$ root $\tilde{\alpha}$, we have $\left\langle\tilde{\alpha}^{\vee}, \tau(\Psi(\lambda))\right\rangle<0$ only if the $\tilde{\alpha}$-root space lies in $\operatorname{Lie}\left(\mathbb{U}\left(\mathfrak{n}^{-}\right)\right)$.

(ii) Let $\lambda$ be a dominant $\mathrm{Sp}_{2 m}$-weight, let $\alpha=\alpha$, be a positive $\mathrm{Sp}_{2 m}$-root, and let $\tilde{\alpha}=\tilde{\alpha}_{j, i+2 m}$ be the $\mathrm{Sp}_{2(2 m)}$ positive root associated with $-\alpha$. Then

$$
\left\langle\alpha^{\vee}, \lambda\right\rangle=-\left\langle\tilde{\alpha}^{\vee}, \bar{\tau}(\Psi(\lambda))\right\rangle .
$$

(iii) Let $\lambda$ be a dominant weight for $\mathrm{Sp}_{2 m}(\mathbb{C})$ and let $\tilde{\alpha}$ be a positive $\mathrm{Sp}_{2(2 m)}$-root. Then $E_{\tilde{\alpha}} v_{\bar{\tau}} \neq 0$ in $\tilde{V}(\Psi(\lambda))$ only if $\tilde{\alpha}=\tilde{\alpha}_{j, i+2 m}$, where $\alpha_{i, j}$ is a positive $\mathrm{Sp}_{2 m}$ root such that $\left\langle\alpha_{i, j}^{\vee}, \lambda\right\rangle>0$.

Proof. Lemma 5.4 implies that for $\lambda=b_{1} \epsilon_{1}+\cdots+b_{m-1} \epsilon_{m-1}$,

$$
\left\langle\left(\tilde{\epsilon}_{i}-\tilde{\epsilon}_{j}\right)^{\vee}, \bar{\tau}(\Psi(\lambda))\right\rangle=\left\{\begin{array}{cl}
b_{i}-b_{j} \geq 0 & \text { if } 1 \leq i<j \leq m, \\
b_{i}+b_{2 m-j+1} \geq 0 & \text { if } 1 \leq i \leq m<j \leq 2 m, \\
-b_{2 m-i+1}+b_{2 m-j+1} \geq 0 & \text { if } m<i<j \leq 2 m,
\end{array}\right.
$$

and

$$
\left\langle\left(\tilde{\epsilon}_{i}+\tilde{\epsilon}_{j-2 m}\right)^{\vee}, \bar{\tau}(\Psi(\lambda))\right\rangle=\left\{\begin{array}{cc}
b_{i}+b_{j-2 m} \geq 0 & \text { if } 1 \leq i \leq m \\
& \text { and } 2 m<j \leq 3 m, \\
b_{i}-b_{4 m-j+1} \geq 0 & \text { if } 1 \leq i \leq m \\
& \text { and } 3 m<j \leq 4 m-i+1, \\
-b_{i}+b_{4 m-j+1} \geq 0 & \text { if } 1 \leq i \leq m, 3 m<j, \\
& \text { and } 4 m-i+1<j, \\
b_{2 m-i+1}+b_{2 m-j+1} \geq 0 & \text { if } m<i \leq 2 m \\
& \text { and } 3 m<j,
\end{array}\right.
$$

where always $i+j \leq 4 m$. This proves the corollary.

\section{The fundamental representations: the $\mathfrak{s p}_{2 m}$ case}

Let $\mathfrak{n}_{\mathbb{Z}}^{-, a, i}$ be the direct sum of all root spaces of Lie $\left(\operatorname{Sp}_{2(2 m)}\right)_{\mathbb{Z}}$ corresponding to positive roots $\beta$ such that $\left\langle\beta^{\vee}, \bar{\tau}\left(\tilde{\omega}_{2 i}\right)\right\rangle<0$ for all $1 \leq i \leq m$. By Lemma 5.5, such a space lies in $\operatorname{Lie}\left(\mathbb{U}\left(\mathfrak{n}_{\mathbb{Z}}^{-}\right)\right)$.

By [Mathieu 1989, Lemme 26], the Demazure module $\tilde{V}_{\mathbb{Z}}\left(\ell \tilde{\omega}_{2 i}\right)_{\tau}$ is isomorphic to the algebra $U_{\mathbb{Z}}(\tilde{\mathfrak{n}})$ modulo the left ideal $\tilde{I}_{\mathbb{Z}}\left(\bar{\tau} \ell \tilde{\omega}_{2 i}\right)$ generated for all $m \geq 1$ by the

$$
\begin{cases}E_{k, l}^{(m)} & \text { if }\left\langle\tilde{\alpha}_{k, l}^{\vee}, \bar{\tau} \tilde{\omega}_{2 i}\right\rangle \geq 0, \\ E_{k, l}^{\left(-\left\langle\tilde{\alpha}_{k, l}^{\vee}, \bar{\tau} \ell \tilde{\omega}_{2 i}\right\rangle+m\right)} & \text { otherwise. }\end{cases}
$$

Therefore all the root vectors (and their divided powers) not lying in $\mathfrak{n}_{\mathbb{Z}}^{-, a, i}$ act trivially on $\tilde{V}_{\mathbb{Z}}\left(\ell \tilde{\omega}_{2 i}\right)_{\bar{\tau}}$ and hence in order to describe its structure as an $\mathfrak{n}_{\mathbb{Z}}^{-, a}$-module it suffices to consider only the $\mathfrak{n}_{\mathbb{Z}}^{-, a, i}$-action. Recall that $v_{\bar{\tau}}=\bar{\tau}\left(v_{0}\right)$ denotes the 
generator of $\tilde{V}_{\mathbb{Z}}\left(\Psi\left(\ell \omega_{i}\right)\right)_{\bar{\tau}}$. Then the above discussion can be summarized as

$$
\tilde{V}_{\mathbb{Z}}\left(\ell \tilde{\omega}_{2 i}\right)_{\bar{\tau}}=U_{\mathbb{Z}}(\tilde{\mathfrak{n}}) \cdot v_{\bar{\tau}}=U_{\mathbb{Z}}\left(\mathfrak{n}^{-, a}\right) \cdot v_{\bar{\tau}}=U_{\mathbb{Z}}\left(\mathfrak{n}^{-, a, i}\right) \cdot v_{\bar{\tau}} .
$$

Recall that we embed in $\left(\mathrm{SL}_{2(2 m)}\right)_{\mathbb{Z}}$ a copy $L(i)_{\mathbb{Z}}$ of $\left(\mathrm{SL}_{2 m}\right)_{\mathbb{Z}}$ so that we can identify the $\mathrm{SL}_{2(2 m)}$-Demazure module $\tilde{V}_{\mathbb{Z}}^{\mathrm{SL}_{2(2 m)}}\left(\ell \tilde{\omega}_{2 i}\right)_{\tau}$ generated by $\tau\left(v_{0}\right)=\bar{\tau}\left(v_{0}\right)=v_{\bar{\tau}}$ with the Weyl module $V_{\mathbb{Z}}^{L(i)}\left(\ell \varpi_{i}\right)$ for $L(i)_{\mathbb{Z}}$.

For $1 \leq k, l \leq 4 m$, denote by $X_{k, l}$ the $4 m \times 4 m$-matrix having a 1 in position $(k, l)$ and whose all other entries 0 (for $k \neq l$, this is the $\mathrm{SL}_{2(2 m)}$-root operator corresponding to the $\mathrm{SL}_{2(2 m)}$-root $\left.\alpha_{k, l}\right)$, so that

$$
\mathfrak{n}^{-, a, i}=\operatorname{span}\left\{X_{r, s}+X_{4 m-s+1,4 m-r+1} \mid i+1 \leq r \leq 2 m \text { and } 2 m<s \leq 2 m+i\right\} .
$$

It is then immediate:

Lemma 6.1. Every element $y \in \mathfrak{n}_{\mathbb{Z}}^{-, a, i}$ can be written in a unique way as $y=y_{1}+y_{2}$, with $y_{1} \in \mathfrak{n}^{-, a, i} \cap \operatorname{Lie} L(i)_{\mathbb{Z}}$ and $y_{2} \in \operatorname{span}\left\{X_{k, l} \mid l>2 m+k\right\}$. Moreover, $y_{2}$ is uniquely determined by $y_{1}$.

By the previous lemma, the projection $p:\left(\mathrm{SL}_{2(2 m)}\right)_{\mathbb{Z}} \rightarrow L(i)_{\mathbb{Z}}$ induces an isomorphism of vector spaces $\mathfrak{n}_{\mathbb{Z}}^{-, a, i} \simeq p\left(\mathfrak{n}_{\mathbb{Z}}^{-, a, i}\right)$. Let us write $\overline{\mathfrak{n}}_{\mathbb{Z}}^{-, a, i}$ for $p\left(\mathfrak{n}^{-, a, i}\right)$. Since the Lie algebras are commutative, we see that $p: \mathfrak{n}_{\mathbb{Z}}^{-, a, i} \rightarrow \overline{\mathfrak{n}}_{\mathbb{Z}}^{-, a, i}$ is not only an isomorphism of vector spaces, but it is in fact a Lie algebra isomorphism.

\section{Corollary 6.2. $\quad \tilde{V}_{\mathbb{Z}}\left(\Psi\left(\ell \omega_{i}\right)\right)_{\bar{\tau}}=U_{\mathbb{Z}}\left(\overline{\mathfrak{n}}^{-, a, i}\right) \cdot v_{\bar{\tau}}$.}

Proof. Let $y \in \mathfrak{n}_{\mathbb{Z}}^{-, a, i}$. By Lemma 6.1 we can write $y=p(y)+y_{2}$ with $y_{2}$ in the span of the matrices $X_{i, j}$ with $j>2 m+i$. Therefore, $y_{2}$ acts trivially on $\tilde{V}_{\mathbb{Z}}\left(\Psi\left(\ell \omega_{i}\right)\right)_{\bar{\tau}}$, and we conclude

$$
\left.\tilde{V}_{\mathbb{Z}}\left(\Psi\left(\ell \omega_{i}\right)\right)_{\bar{\tau}}\right)=U_{\mathbb{Z}}\left(\mathfrak{n}^{-, a, i}\right) \cdot v_{\bar{\tau}}=U_{\mathbb{Z}}\left(\overline{\mathfrak{n}}^{-, a, i}\right) \cdot v_{\bar{\tau}} .
$$

For $0 \leq i \leq m-1$, let $\operatorname{Sp}_{2 m}(i)_{\mathbb{Z}}$ be a copy of $\left(\operatorname{Sp}_{2 m}\right)_{\mathbb{Z}}$ sitting inside $L(i)_{\mathbb{Z}}$, defined with respect to the form given by the matrix

$$
\left(\begin{array}{cccc}
0 & J_{m-i} & 0 & 0 \\
-J_{m-i} & 0 & 0 & 0 \\
0 & 0 & 0 & J_{i} \\
0 & 0 & -J_{i} & 0
\end{array}\right)
$$

where $J_{r}$ denotes the $r \times r$ antidiagonal matrix with entries $(1,1, \ldots, 1)$. Moreover, denote $\left(\mathrm{Sp}_{2 m}^{\text {std }}\right)_{\mathbb{Z}}:=\mathrm{Sp}_{2 m}(0)_{\mathbb{Z}}$.

Let $\sigma$ be the permutation in $W^{L(i)}$ such that $\operatorname{Lie} \operatorname{Sp}_{2 m}(i)_{\mathbb{Z}}=\sigma \operatorname{Lie}\left(\operatorname{Sp}_{2 m}^{\text {std }}\right)_{\mathbb{Z}} \sigma^{-1}$. The permutation $\sigma$ fixes $2 m+1, \ldots, 2 m+i$ and moves $2 m-i+1, \ldots, 2 m$ in 
front of $i+1, \ldots, 2 m-i$, so that

$$
\begin{aligned}
\sigma\left(e_{1} \wedge e_{2} \wedge \cdots \wedge e_{i} \wedge e_{2 m+1}\right. & \left.\wedge e_{2 m+2} \wedge \cdots \wedge e_{2 m+i}\right) \\
=e_{1} & \wedge e_{2} \wedge \cdots \wedge e_{i} \wedge e_{2 m+1} \wedge e_{2 m+2} \wedge \cdots \wedge e_{2 m+i}
\end{aligned}
$$

Let $\mathfrak{b}_{\mathbb{Z}} \subset \operatorname{Lie}\left(\mathrm{Sp}_{2 m}^{\text {std }}\right)_{\mathbb{Z}}$ be the Borel subalgebra of upper triangular matrices. Let $\mathfrak{p}_{\mathbb{Z}}^{i} \subset \operatorname{Lie}\left(\operatorname{Sp}_{2 m}^{\text {std }}\right)_{\mathbb{Z}}$ be the maximal parabolic subalgebra associated with $\varpi_{i}$, and $\mathfrak{p}_{\mathbb{Z}}^{i, n}$ its nilpotent radical. Write $S \mathfrak{p}_{\mathbb{Z}}^{i, n}$ for $\sigma \mathfrak{p}_{\mathbb{Z}}^{i, n} \sigma^{-1} \subset \operatorname{Lie} \operatorname{Sp}_{2 m}(i)_{\mathbb{Z}}$.

\section{Lemma 6.3. $\quad V_{\mathbb{Z}}\left(\ell \varpi_{i}\right)=U_{\mathbb{Z}}\left(S \mathfrak{p}^{i, n}\right) \cdot v_{\bar{\tau}}$.}

Proof. By (6), $v_{\bar{\tau}}$ is a lowest weight vector for $\operatorname{Sp}_{2 m}(i)$, as well as for $L(i)$, and the module generated by this vector is $U_{\mathbb{Z}}\left(\operatorname{Lie~Sp}_{2 m}(i)\right) \cdot v_{\bar{\tau}}=V_{\mathbb{Z}}\left(\ell \varpi_{i}\right)$. Since it is generated by a lowest weight vector, it is enough to consider the action of the nilpotent radical

$$
U_{\mathbb{Z}}\left(\operatorname{Lie~} \operatorname{Sp}_{2 m}(i)\right) \cdot v_{\bar{\tau}}=U_{\mathbb{Z}}\left(\sigma \mathfrak{b} \sigma^{-1}\right) \cdot v_{\bar{\tau}}=U_{\mathbb{Z}}\left(S \mathfrak{p}^{i, n}\right) \cdot v_{\bar{\tau}} .
$$

Observe that since $\overline{\mathfrak{n}}_{\mathbb{Z}}^{-, a, i} \subseteq S \mathfrak{p}_{\mathbb{Z}}^{i, n}$, the Weyl module $V_{\mathbb{Z}}\left(\ell \varpi_{i}\right)$ is naturally equipped with a structure of $\overline{\mathfrak{n}}^{-, a, i}$-module. It is easy to check that:

Lemma 6.4. Every element $x \in S \mathfrak{p}_{\mathbb{Z}}^{i, n}$ can be written in a unique way as $x=x_{1}+x_{2}$, with $x_{1} \in \overline{\mathfrak{n}}^{-, a, i}$ and $x_{2} \in \operatorname{span}\left\{X_{k, l} \mid 2 m-i<k \leq 2 m\right.$ and $\left.i+1<l<2 m\right\}$. Moreover, $x_{2}$ is uniquely determined by $x_{1}$.

As in Section 3, we consider the Chevalley involution $\iota: \mathfrak{s p}_{2 m} \rightarrow \mathfrak{s p}_{2 m}$ such that $\iota l_{\mathfrak{h}}=-1$ and $\iota$ exchanges $e_{\alpha}$ and $-f_{\alpha}$. It induces an isomorphism $S_{\mathbb{Z}}^{\bullet}\left(\mathfrak{n}^{-}\right) \rightarrow S_{\mathbb{Z}}^{\bullet}\left(\mathfrak{n}^{+}\right)$, which by abuse we also call $\iota$.

For a dominant weight $\lambda$, fix a highest weight vector $v_{\lambda} \in V_{\mathbb{Z}}(\lambda)$ and a lowest weight vector $v_{w_{0}} \in V_{\mathbb{Z}}(\lambda)$, where $w_{0}$ is the longest word in the Weyl group of $\mathfrak{s p}_{2 m}$. Recall that considering the PBW filtration on $U_{\mathbb{Z}}\left(\mathfrak{n}^{-}\right)$and on $U_{\mathbb{Z}}\left(\mathfrak{n}^{+}\right)$provides $V_{\mathbb{Z}}(\lambda)$ with two possible $S_{\mathbb{Z}}^{\bullet}\left(\mathfrak{n}^{-, a}\right)$-structures: in the first case, looking at the PBW filtration on $V_{\mathbb{Z}}(\lambda)$ induced by the action of $U_{\mathbb{Z}}\left(\mathfrak{n}^{-}\right)$on the highest weight vector and taking the associated graded space provides the abelianized module $V_{\mathbb{Z}}^{a}(\lambda)$, while in the second case, looking at the PBW filtration on $V_{\mathbb{Z}}(\lambda)$ induced by the action of $U_{\mathbb{Z}}\left(\mathfrak{n}^{+}\right)$on the lowest weight vector and taking the associated graded space produces a module that we denote by $V_{\mathbb{Z}}^{a,+}(\lambda)$. Now via $\iota$ this module also becomes naturally a $S_{\mathbb{Z}}^{\bullet}\left(\mathfrak{n}^{-}\right)$-module and Lemma 3.3 holds in the symplectic case too:

Lemma 6.5. As a $S_{\mathbb{Z}}^{\bullet}\left(\mathfrak{n}^{-}\right)$-module, $V_{\mathbb{Z}}^{a,+}(\lambda)$ is isomorphic to $V_{\mathbb{Z}}^{a}(\lambda)$.

Observe that in the symplectic case there is no need of replacing $\lambda$ by $\lambda^{*}$ since they coincide.

Lemma 6.6. The Demazure module $\tilde{V}_{\mathbb{Z}}\left(\Psi\left(\ell \omega_{i}\right)\right)_{\bar{\tau}}$ contained in $\tilde{V}_{\mathbb{Z}}\left(\Psi\left(\ell \omega_{i}\right)\right)$ and $V_{\mathbb{Z}}\left(\ell \omega_{i}\right)^{a}$ are isomorphic as $S_{\mathbb{Z}}^{\cdot}\left(\mathfrak{n}^{-}\right)$-modules. 
Proof. By Lemma 6.4, the projection $q: S \mathfrak{p}_{\mathbb{Z}}^{i, n} \rightarrow \overline{\mathfrak{n}}^{-, a, i}$ is an isomorphism of vector spaces. Moreover, if we write $x \in S \mathfrak{p}_{\mathbb{Z}}^{i, n}$ as $x=q(x)+x_{2}$, then $x_{2}$ lies in the span of the matrices $X_{i, j}$ with $i+1<j<2 m$; hence, $x_{2} \cdot v_{\bar{\tau}}=0$. Now, by [Feigin et al. 2014, Proposition 3.1], the PBW filtrations on $V_{\mathbb{Z}}\left(\ell \omega_{i}\right)$ with respect to the actions of $S \mathfrak{p}_{\mathbb{Z}}^{i, n}$ and $\overline{\mathfrak{n}}_{\mathbb{Z}}^{-, a, i}$ are compatible, and

$$
\operatorname{gr} V_{\mathbb{Z}}\left(\ell \varpi_{i}\right)=\operatorname{gr} U_{\mathbb{Z}}\left(S \mathfrak{p}_{\mathbb{Z}}^{i, n}\right) \cdot v_{\bar{\tau}} \simeq \operatorname{gr} U_{\mathbb{Z}}\left(\overline{\mathfrak{n}}^{-, a, i}\right) \cdot v_{\bar{\tau}} .
$$

On the other hand, when we consider in $\tilde{V}_{\mathbb{Z}}\left(\Psi\left(\ell \omega_{i}\right)\right)_{\bar{\tau}}$ the PBW filtration with respect to the action of $S \mathfrak{p}_{\mathbb{Z}}^{i, n}$ and go to the associated graded module, then the action of $\left(S \mathfrak{p}_{\mathbb{Z}}^{i, n}\right)^{a}$ is isomorphic to the action of $\overline{\mathfrak{n}}_{\mathbb{Z}}^{-, a, i}$ on $\tilde{V}_{\mathbb{Z}}\left(\Psi\left(\ell \omega_{i}\right)\right)_{\bar{\tau}}$.

The previous result implies in particular:

Corollary 6.7.

$\operatorname{rank} \tilde{V}_{\mathbb{Z}}\left(\Psi\left(\ell \omega_{i}\right)\right)_{\bar{\tau}}=\operatorname{rank} V_{\mathbb{Z}}\left(\ell \omega_{i}\right)$.

\section{The general case for $\mathfrak{s p}_{2 m}$}

We come now to the general case (notation as in Example 1.2):

Theorem 7.1. Let $\lambda$ be a dominant $\mathfrak{s p}_{2 m}$-weight. As an $N_{\mathbb{Z}, \eta}^{-, a}$-module, the Demazure submodule $\tilde{V}_{\mathbb{Z}}(\Psi(\lambda))_{\bar{\tau}}$ of the $\left(\operatorname{Sp}_{2(2 m)}\right)_{\mathbb{Z}}$-module $\tilde{V}_{\mathbb{Z}}(\Psi(\lambda))$ is isomorphic to the abelianized module $V_{\mathbb{Z}}^{a}(\lambda)$.

As in the type A case, the proof of the above theorem will provide us with a description of $V_{\mathbb{Z}}^{a}(\lambda)$ as an $S_{\mathbb{Z}}^{\bullet}\left(\mathfrak{n}_{\eta}^{-, a}\right)$-module in terms of generators and relations. The abelianized module $V_{\mathbb{Z}}^{a}(\lambda)$ is a cyclic module over the algebra $S_{\mathbb{Z}}^{\bullet}\left(\mathfrak{n}_{\eta}^{-, a}\right)$ with the image of a highest weight vector $v_{\lambda} \in V(\lambda)$ in $V_{\mathbb{Z}}^{a}(\lambda)$ as a generator; see [Feigin et al. 2013, Proposition 2.3]. Hence the module is isomorphic to $S_{\mathbb{Z}}^{\bullet}\left(\mathfrak{n}_{\eta}^{-, a}\right) / I_{\mathbb{Z}}(\lambda)$ where $I_{\mathbb{Z}}(\lambda)$ is the annihilator of $v_{\lambda}$ in $S_{\mathbb{Z}}\left(\mathfrak{n}_{\eta}^{-, a}\right)$. As a consequence of the proof of Theorem 7.1, we obtain the description of the ideal $I_{\mathbb{Z}}(\lambda)$ in terms of generators given in [Feigin et al. 2011a; 2013] from Mathieu's generator and relation presentation of Demazure modules.

Let $\mathfrak{b}$ be the Borel subalgebra of $\mathfrak{s p}_{2 m}=\left(\mathfrak{s p}_{2 m}\right)_{\mathbb{Z}} \otimes \mathbb{C}$ as in Example 1.1(b), so $\mathfrak{s p}_{2 m}=\mathfrak{n}^{-} \oplus \mathfrak{h} \oplus \mathfrak{n}^{+}$. As free $\mathbb{Z}$-modules, $U_{\mathbb{Z}}\left(\mathfrak{n}^{-}\right) \simeq U_{\mathbb{Z}}(\mathfrak{g}) / U_{\mathbb{Z}}^{+}\left(\mathfrak{h}+\mathfrak{n}^{+}\right)$, so that the adjoint action of $U_{\mathbb{Z}}(\mathfrak{b})$ on $U_{\mathbb{Z}}(\mathfrak{g})$ induces the structures of a $U_{\mathbb{Z}}^{+}(\mathfrak{b})$ - and

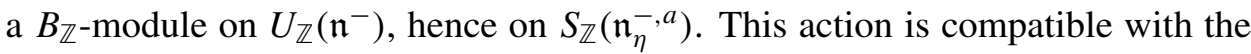

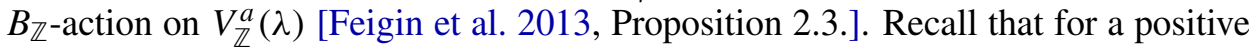
root $\alpha$ we have denoted by $f_{\alpha}$ the corresponding fixed Chevalley basis element in

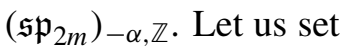

$$
R^{++}=\left\{\epsilon_{i}-\epsilon_{j} \mid 1 \leq i<j \leq m\right\} \cup\left\{2 \epsilon_{i} \mid 1 \leq i \leq m\right\}
$$

As a consequence of the proof of Theorem 7.1 we get the following description of the ideal $I_{\mathbb{Z}}(\lambda)$ : 
Corollary 7.2. As a cyclic $S_{\mathbb{Z}}^{\bullet}\left(\mathfrak{n}_{\eta}^{-, a}\right)$-module, the abelianized module $V_{\mathbb{Z}}^{a}(\lambda)$ is isomorphic to $S_{\mathbb{Z}}^{\bullet}\left(\mathfrak{n}_{\eta}^{-, a}\right) / I_{\mathbb{Z}}(\lambda)$, where

$I_{\mathbb{Z}}(\lambda)=S_{\mathbb{Z}}^{\bullet}\left(\mathfrak{n}_{\eta}^{-, a}\right)\left(U_{\mathbb{Z}}\left(\mathfrak{n}^{+}\right) \circ \operatorname{span}\left\{f_{\alpha}^{\left(\left\langle\lambda, \alpha^{\vee}\right\rangle+m\right)} \mid m \geq 1\right.\right.$ and $\left.\left.\alpha \in R^{++}\right\}\right) \subseteq S_{\mathbb{Z}}^{\bullet}\left(\mathfrak{n}_{\eta}^{-, a}\right)$.

7A. The proof of the theorem will be only sketched, since the strategy is the same as for the type A case. We reproduce here the diagram of $S^{\bullet}\left(\mathfrak{n}_{\eta}^{-, a}\right)$-modules summarizing the main idea: for a dominant weight $\lambda=a_{1} \omega_{1}+\cdots+a_{m} \omega_{m}$, we have the natural maps

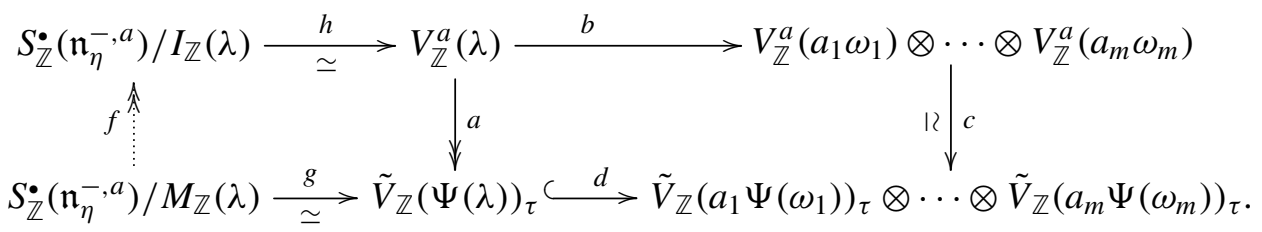

where in the top row the action on the modules is twisted by the Chevalley involution so the cyclic generators are lowest weight vectors, and the maps $c, d, a$, and $g$ arise as in the proof of Theorem 4.1 so that again the main difficulty of the proof consists in producing the map $f$.

7B. The first step consists in determining $M_{\mathbb{Z}}(\lambda)$. By [Mathieu 1989, Lemme 26], the Demazure module $\tilde{V}_{\mathbb{Z}}(\Psi(\lambda))_{\tau}$ is isomorphic to the algebra $U_{\mathbb{Z}}(\tilde{\mathfrak{n}})$ modulo the left ideal $\tilde{I}_{\mathbb{Z}}(\tau \Psi(\lambda))$ generated for all $m \geq 1$ by the elements

$$
\begin{cases}E_{k, l}^{(m)} & \text { if }\left\langle\tilde{\alpha}_{k, l}^{\vee}, \tau \Psi(\lambda)\right\rangle \geq 0, \\ E_{k, l}^{\left(-\left\langle\tilde{\alpha}_{k, l}^{\vee}, \tau \Psi(\lambda)\right\rangle+m\right)} & \text { otherwise. }\end{cases}
$$

7C. The annihilator $M_{\mathbb{Z}}(\lambda)$ is the intersection of $U_{\mathbb{Z}}\left(\mathfrak{n}_{\eta}^{-, a}\right) \subset U_{\mathbb{Z}}\left(\tilde{\mathfrak{n}}^{+}\right)$with the ideal $\tilde{I}_{\mathbb{Z}}(\bar{\tau} \Psi(\lambda))$. To determine such an intersection, we fix a PBW basis and divide the positive roots in three families, exactly as in the proof of Theorem 4.1.

7D. By Lemma 5.5(i), $\left\langle\tilde{\alpha}_{k, l}^{\vee}, \tau \Psi(\lambda)\right\rangle \geq 0$ if $\tilde{\alpha}_{k, l}$ is of third type. As in type A, we may hence proceed with the calculation modulo the left ideal generated by the divided powers of the corresponding $E_{k, l}$. Modulo such an ideal, by Lemma 5.4 and Lemma 5.5, $\tilde{I}_{\mathbb{Z}}(\bar{\tau} \Psi(\lambda))$ is generated by the $E_{k, l}^{(m)}$ with $m \geq 1$ and $\tilde{\alpha}_{k, l}$ of second type, and the

$$
E_{k, l}^{\left(-\left\langle\tilde{\alpha}_{k, l}^{\vee}, \Psi(\lambda)\right\rangle+m\right)} \quad \text { with } m \geq 1 \text { and } \tilde{\alpha}_{k, l} \text { of first type. }
$$

7E. Thus, we consider the subalgebra a generated by the $E_{k, l}^{(m)}$, for $\tilde{\alpha}_{k, l}$ of second type. Let $\mathfrak{b}_{\mathbb{Z}, \mathfrak{s l}_{2 m}}$ be the Borel subalgebra of $\mathfrak{s l}_{2 m}$ consisting of traceless upper triangular matrices and let $\mathfrak{b}_{\mathbb{Z}}$ be the corresponding symplectic Borel subalgebra (of $\left.\left(\mathrm{Sp}_{2 m}^{\text {std }}\right)_{\mathbb{Z}}\right)$. Let us embed $\mathfrak{b}_{\mathbb{Z}}$ in $\mathfrak{b}_{\mathbb{Z}, \mathfrak{s} \mathfrak{L}_{2 m}} \oplus \mathfrak{b}_{\mathbb{Z}, \mathfrak{s} \mathfrak{s}_{2 m}}$ via $A \mapsto(A,-\bar{A})$, where $\bar{A}$ denotes the matrix which is skew-transposed to $A$, and let $\Delta^{-}\left(\mathfrak{b}_{\mathbb{Z}}\right)$ be its image. Also $\mathfrak{a}$ 
is embedded in $\mathfrak{b}_{\mathbb{Z}, \mathfrak{s}_{2 m}} \oplus \mathfrak{b}_{\mathbb{Z}, \mathfrak{s} \mathfrak{l}_{2 m}}$, once we identify the latter with the Lie algebra generated by the divided powers of the $\mathrm{SL}_{4 m}$-root vectors of second type. The image of such an embedding contains $\Delta^{-}\left(\mathfrak{b}_{\mathbb{Z}}\right)$. By taking fixed points with respect to the outer automorphism of $\mathfrak{s l}_{2 m}$ and $\mathfrak{s l}_{4 m}$ induced by the symmetry of the Dynkin diagram, it follows from Lemma 4.5 that

$$
U_{\mathbb{Z}}\left(\Delta^{-}\left(\mathfrak{b}_{\mathbb{Z}}\right)\right)\left\langle\left\{E_{i, j}^{(m)} \mid \alpha_{i, j} \text { of first type, } m \geq 1\right\}\right\rangle
$$

Therefore,

$$
=U_{\mathbb{Z}}(\mathfrak{a})\left\langle\left\{E_{i, j}^{(m)} \mid \alpha_{i, j} \text { of first type, } m \geq 1\right\}\right\rangle .
$$

$$
\begin{aligned}
& \tilde{I}_{\mathbb{Z}}(\tau \Psi(\lambda)) \cap S_{\mathbb{Z}}^{\bullet}\left(\mathfrak{n}_{\eta}^{-, a}\right) \\
& \quad \simeq S_{\mathbb{Z}}^{\bullet}\left(\mathfrak{n}_{\eta}^{-, a}\right) \circ U_{\mathbb{Z}}\left(\Delta^{-}\left(\mathfrak{b}_{\mathbb{Z}}\right)\right) \operatorname{span}\left\{f_{i, j}^{\left\langle\alpha^{\vee}, \lambda\right\rangle+\ell} \mid \alpha \in R^{++} \text {and } \ell \geq 1\right\}=: M_{\mathbb{Z}}(\lambda) .
\end{aligned}
$$

7F. Proof of Theorem 7.1. Since the roots of first type are precisely the ones coming from the elements $f_{i, j}$ with $\alpha_{i, j} \in R^{++}$and since

$$
\left\{f_{i, j}^{\left(\left\langle\alpha_{i, j}^{\vee}, \lambda\right\rangle+m\right)} \mid \alpha_{i, j} \in R^{++} \text {and } m \geq 1\right\} \subseteq I_{\mathbb{Z}}(\lambda),
$$

we get a surjective morphism

$$
\tilde{V}_{\mathbb{Z}}(\Psi \lambda)_{\bar{\tau}} \stackrel{g}{\simeq} S_{\mathbb{Z}}^{\bullet}\left(\mathfrak{n}_{\eta}^{-, a}\right) / M_{\mathbb{Z}}(\lambda) \stackrel{f}{\rightarrow} S_{\mathbb{Z}}^{\bullet}\left(\mathfrak{n}_{\eta}^{-, a}\right) / I_{\mathbb{Z}}(\lambda) \simeq V_{\mathbb{Z}}(\lambda)^{a} .
$$

This concludes the proof of the theorem.

7G. Let $\rho$ be the sum of the fundamental weights for $\mathrm{Sp}_{2 m}$ and let $\tilde{\rho}=\Psi(\rho)$ be the corresponding dominant weight for $\mathrm{Sp}_{2(2 m)}$. Let $Q_{\mathbb{Z}} \subset\left(\operatorname{Sp}_{2(2 m)}\right)_{\mathbb{Z}}$ be the corresponding parabolic subgroup. Recall that $N_{\mathbb{Z}, \eta}^{-, a}$ is a commutative subgroup of the Borel subgroup $\tilde{B}_{\mathbb{Z}}$. For any $\operatorname{Sp}_{2(2 m)}$-root $\tilde{\alpha}$, let $U_{\mathbb{Z}, \tilde{\alpha}}$ be the associated root subgroup.

Lemma 7.3. The orbit $\tilde{B}_{\mathbb{Z}} \cdot \bar{\tau} \subset\left(\operatorname{Sp}_{2(2 m)}\right)_{\mathbb{Z}} / Q_{\mathbb{Z}}$ is nothing but $N_{\mathbb{Z}, \eta}^{-, a} \cdot \bar{\tau}$, and the map $N_{\mathbb{Z}, \eta}^{-, a} \rightarrow N_{\mathbb{Z}, \eta}^{-, a} \cdot \bar{\tau}$, given by $u \mapsto u \bar{\tau}$ is a bijection.

Proof. We have $\tilde{B}_{\mathbb{Z}} \cdot \bar{\tau}=\prod_{\tilde{\alpha}>0} U_{\mathbb{Z}, \alpha} \cdot \bar{\tau}$, and the map $\prod_{\tilde{\alpha} \in \Gamma} U_{\mathbb{Z}, \tilde{\alpha}} \rightarrow \prod_{\tilde{\alpha} \in \Gamma} U_{\mathbb{Z}, \tilde{\alpha}} \cdot \bar{\tau}$ is a bijection, where $\Gamma$ is the set of all positive roots of $\operatorname{Sp}_{2(2 m)}$ such that $\bar{\tau}^{-1}(\tilde{\alpha})<0$ and $\bar{\tau}^{-1}(\tilde{\alpha})$ is not an element of the root system of $Q_{\mathbb{Z}}$. Now this condition is fulfilled if and only if $\left\langle\bar{\tau}^{-1}\left(\tilde{\alpha}^{\vee}\right), \tilde{\rho}\right\rangle<0$, or, equivalently, $\left\langle\tilde{\alpha}^{\vee}, \bar{\tau}(\tilde{\rho})\right\rangle<0$. By Lemma 5.4 this is not possible if $\tilde{\alpha}$ is of the form $\tilde{\alpha}=\tilde{\epsilon}_{i}-\tilde{\epsilon}_{j}$, with $1 \leq i<j \leq 2 m$. For the long roots, this is only possible if $\tilde{\alpha}=2 \tilde{\epsilon}_{j}$, with $j=m+1, \ldots, 2 m$, and for the roots $\alpha=\tilde{\epsilon}_{i}+\tilde{\epsilon}_{j}$, with $1 \leq i<j \leq 2 m$; this is only possible if either $i, j \geq m+1$ or $1 \leq i \leq m$ and $j=2 m+1-k$ is such that $1 \leq k<i$.

But this implies that the root subgroup $U_{\mathbb{Z}, \tilde{\alpha}}$ is a subgroup of $N_{\mathbb{Z}, \eta}^{-, a}$, and all root subgroups of $\left(\mathrm{Sp}_{2(2 m)}\right)_{\mathbb{Z}}$ contained in $N_{\mathbb{Z}, \eta}^{-, a}$ satisfy this condition. It follows that $N_{\mathbb{Z}, \eta}^{-, a} . \bar{\tau}$ is the product of all root subgroups corresponding to positive roots of 
$\operatorname{Sp}_{2(2 m)}$ such that $\bar{\tau}^{-1}(\tilde{\alpha})<0$ and $\bar{\tau}^{-1}(\alpha)$ is not an element of the root system of $Q_{\mathbb{Z}}$ and hence $N_{\mathbb{Z}, \eta}^{-, a} \cdot \bar{\tau}=\tilde{B}_{\mathbb{Z}} \cdot \bar{\tau} \subset\left(\operatorname{Sp}_{2(2 m)}\right)_{\mathbb{Z}} / Q_{\mathbb{Z}}$.

Corollary 7.4. The degenerate flag variety $\mathcal{F} \ell(\lambda)_{k}^{a}$ depends only on supp $\lambda$. It is a projectively normal variety, Frobenius split, with rational singularities.

\section{Acknowledgements}

The work of M. Lanini and P. Littelmann was partially supported by the DFGSchwerpunkt Grant SP1388. The work of G.C.I. was financed by the national FIRB grant RBFR12RA9W "Perspectives in Lie Theory". We thank Corrado De Concini for many helpful conversations about this project.

\section{References}

[Bourbaki 1968] N. Bourbaki, Groupes et algèbres de Lie: Chapitres 4, 5, 6, Hermann, Paris, 1968. MR 0240238

[Cerulli Irelli and Lanini 2015] G. Cerulli Irelli and M. Lanini, "Degenerate flag varieties of type A and C are Schubert varieties", Int. Math. Res. Not. 2015:15 (2015), 6353-6374. MR 3384481

[Feigin 2012] E. Feigin, " $\mathbb{G}_{a}^{M}$ degeneration of flag varieties", Selecta Math. (N.S.) 18:3 (2012), 513-537. MR 2960025

[Feigin and Finkelberg 2013] E. Feigin and M. Finkelberg, "Degenerate flag varieties of type A: Frobenius splitting and BW theorem”, Math. Z. 275:1-2 (2013), 55-77. MR 3101796

[Feigin et al. 2011a] E. Feigin, G. Fourier, and P. Littelmann, "PBW filtration and bases for irreducible modules in type $\mathrm{A}_{n}$ ", Transform. Groups 16:1 (2011), 71-89. MR 2785495

[Feigin et al. 2011b] E. Feigin, G. Fourier, and P. Littelmann, "PBW filtration and bases for symplectic Lie algebras", Int. Math. Res. Not. 2011:24 (2011), 5760-5784. MR 2863380

[Feigin et al. 2013] E. Feigin, G. Fourier, and P. Littelmann, "PBW-filtration over $\mathbb{Z}$ and compatible bases for $V_{\mathbb{Z}}(\lambda)$ in type $A_{n}$ and $C_{n}$ ", pp. 35-63 in Symmetries, integrable systems and representations (Tokyo, 2011 and Lyon, 2011), edited by K. Iohara et al., Springer Proc. Math. Stat. 40, Springer, Heidelberg, 2013. MR 3077680

[Feigin et al. 2014] E. Feigin, M. Finkelberg, and P. Littelmann, "Symplectic degenerate flag varieties", Canad. J. Math. 66:6 (2014), 1250-1286. MR 3270783

[Jantzen 1987] J. C. Jantzen, Representations of algebraic groups, Pure and Applied Mathematics 131, Academic Press, Boston, 1987. MR 899071

[Lakshmibai et al. 1979] V. Lakshmibai, C. Musili, and C. S. Seshadri, "Geometry of G/P", Bull. Amer. Math. Soc. (N.S.) 1:2 (1979), 432-435. MR 520081

[Mathieu 1989] O. Mathieu, "Construction d'un groupe de Kac-Moody et applications", Compositio Math. 69:1 (1989), 37-60. MR 986812

[Mehta and Ramanathan 1988] V. B. Mehta and A. Ramanathan, "Schubert varieties in $G / B \times G / B$ ", Compositio Math. 67:3 (1988), 355-358. MR 959217

[Ramanan and Ramanathan 1985] S. Ramanan and A. Ramanathan, "Projective normality of flag varieties and Schubert varieties", Invent. Math. 79:2 (1985), 217-224. MR 778124

[Ramanathan 1987] A. Ramanathan, "Equations defining Schubert varieties and Frobenius splitting of diagonals", Inst. Hautes Études Sci. Publ. Math. 65 (1987), 61-90. MR 908216 
Received November 16, 2015. Revised May 6, 2016.

Giovanni CERUlli Irelli

DiPARTIMENTO DI MATEMATICA

SAPIENZA UNIVERSITÀ DI ROMA

PIAZZALE ALDo Moro 5

I-00185 ROMA

ITALY

cerulli@mat.uniroma1.it

cerulli.math@googlemail.com

MARTINA LANINI

SCHOOL OF MATHEMATICS

UNIVERSITY OF EDINBURGH

JAMES Clerk MaXWELl BuILding

Peter Guthrie Tait RoAd

EDINBURGH

EH9 3FD

UNITED KINGDOM

m.lanini@ed.ac.uk

Peter LitTelmanN

Mathematical Institute

UNIVERSITY OF COLOGNE

WEYERTAL 86-90

D-50931 COLOGNE

GERMANY

peter.littelmann@math.uni-koeln.de 


\title{
PACIFIC JOURNAL OF MATHEMATICS
}

Founded in 1951 by E. F. Beckenbach (1906-1982) and F. Wolf (1904-1989)

$$
\text { msp.org/pjm }
$$

\section{EDITORS}

\author{
Don Blasius (Managing Editor) \\ Department of Mathematics \\ University of California \\ Los Angeles, CA 90095-1555 \\ blasius@math.ucla.edu
}

\author{
Paul Balmer \\ Department of Mathematics \\ University of California \\ Los Angeles, CA 90095-1555 \\ balmer@math.ucla.edu \\ Robert Finn \\ Department of Mathematics \\ Stanford University \\ Stanford, CA 94305-2125 \\ finn@math.stanford.edu \\ Sorin Popa \\ Department of Mathematics \\ University of California \\ Los Angeles, CA 90095-1555 \\ popa@math.ucla.edu
}

\author{
Vyjayanthi Chari \\ Department of Mathematics \\ University of California \\ Riverside, CA 92521-0135 \\ chari@math.ucr.edu \\ Kefeng Liu \\ Department of Mathematics \\ University of California \\ Los Angeles, CA 90095-1555 \\ liu@math.ucla.edu \\ Igor Pak \\ Department of Mathematics \\ University of California \\ Los Angeles, CA 90095-1555 \\ pak.pjm@gmail.com \\ Paul Yang \\ Department of Mathematics \\ Princeton University \\ Princeton NJ 08544-1000 \\ yang@math.princeton.edu
}

\section{PRODUCTION}

Silvio Levy, Scientific Editor, production@msp.org

\section{SUPPORTING INSTITUTIONS}

ACADEMIA SINICA, TAIPEI

CALIFORNIA INST. OF TECHNOLOGY

STANFORD UNIVERSITY

UNIV. OF BRITISH COLUMBIA

UNIV. OF CALIFORNIA, BERKELEY

UNIV. OF CALIFORNIA, DAVIS

UNIV. OF CALIFORNIA, LOS ANGELES

UNIV. OF CALIFORNIA, RIVERSIDE

UNIV. OF CALIFORNIA, SAN DIEGO

UNIV. OF CALIF., SANTA BARBARA
KEIO UNIVERSITY

MATH. SCIENCES RESEARCH INSTITUTE

NEW MEXICO STATE UNIV.

OREGON STATE UNIV.
Daryl Cooper

Department of Mathematics

University of California

Santa Barbara, CA 93106-3080 cooper@math.ucsb.edu

Jiang-Hua Lu

Department of Mathematics

The University of Hong Kong

Pokfulam Rd., Hong Kong

jhlu@maths.hku.hk

$$
\text { Jie Qing }
$$

Department of Mathematics

University of California

Santa Cruz, CA 95064

qing@ cats.ucsc.edu

\author{
UNIV. OF CALIF., SANTA CRUZ \\ UNIV. OF MONTANA \\ UNIV. OF OREGON \\ UNIV. OF SOUTHERN CALIFORNIA \\ UNIV. OF UTAH \\ UNIV. OF WASHINGTON \\ WASHINGTON STATE UNIVERSITY
}

These supporting institutions contribute to the cost of publication of this Journal, but they are not owners or publishers and have no responsibility for its contents or policies.

See inside back cover or msp.org/pjm for submission instructions.

The subscription price for 2016 is US $\$ 440 /$ year for the electronic version, and \$600/year for print and electronic.

Subscriptions, requests for back issues and changes of subscriber address should be sent to Pacific Journal of Mathematics, P.O. Box 4163, Berkeley, CA 94704-0163, U.S.A. The Pacific Journal of Mathematics is indexed by Mathematical Reviews, Zentralblatt MATH, PASCAL CNRS Index, Referativnyi Zhurnal, Current Mathematical Publications and Web of Knowledge (Science Citation Index).

The Pacific Journal of Mathematics (ISSN 0030-8730) at the University of California, c/o Department of Mathematics, 798 Evans Hall \#3840, Berkeley, CA 94720-3840, is published twelve times a year. Periodical rate postage paid at Berkeley, CA 94704, and additional mailing offices. POSTMASTER: send address changes to Pacific Journal of Mathematics, P.O. Box 4163, Berkeley, CA 94704-0163.

PJM peer review and production are managed by EditFLOW ${ }^{\circledR}$ from Mathematical Sciences Publishers.

PUBLISHED BY

\section{I. mathematical sciences publishers}

nonprofit scientific publishing

http://msp.org/

(C) 2016 Mathematical Sciences Publishers 


\section{PACIFIC JOURNAL OF MATHEMATICS}

Volume $284 \quad$ No. $2 \quad$ October 2016

Spherical CR Dehn surgeries

Miguel ACOsta

Degenerate flag varieties and Schubert varieties: a characteristic free approach

283

Giovanni Cerulli Irelli, Martina LANini and Peter

LITTELMANN

Solitons for the inverse mean curvature flow

Gregory Drugan, HoJoo LEE and GLEN WHEELER

Bergman theory of certain generalized Hartogs triangles

\section{LUKE D. EDHOLM}

Transference of certain maximal Hilbert transforms on the torus

DASHAN FAN, HUOXIONG WU and FAYOU ZHAO

The Turaev and Thurston norms

STEFAN FriedL, DANIEL S. Silver and Susan G. WiLLiams

A note on nonunital absorbing extensions

JAMES GABE

On nonradial singular solutions of supercritical biharmonic equations

Zongming GuO, JUNCHENG WeI and Wen YANG

Natural commuting of vanishing cycles and the Verdier dual

\section{DAVID B. MASSEY}

The nef cones of and minimal-degree curves in the Hilbert schemes of points on certain surfaces

ZHENBo QIN and YUPING TU

Smooth approximation of conic Kähler metric with lower Ricci curvature bound

\section{LIANGMING SHEN}

Maps from the enveloping algebra of the positive Witt algebra to regular algebras

Susan J. SierRa and Chelsea Walton 